\title{
Personalidad y trastorno de pánico: un estudio de revisión
}

\author{
Jorge Osma ${ }^{1,2, *}$, Azucena García-Palacios ${ }^{3}$ y Cristina Botella ${ }^{3}$ \\ ${ }^{1}$ Universidad de Zaragoza. Campus de Teruel \\ ${ }^{2}$ Creos, centro de psicoterapia y formación. Castellón \\ ${ }^{3}$ Universitat Jaume I. Castellón
}

\begin{abstract}
Resumen: A través de este trabajo ofreceremos una revisión exhaustiva de las aportaciones científicas que se han realizado en las últimas tres décadas sobre la influencia de la personalidad en aspectos clínicos y psicopatológicos del Trastorno de pánico (TP), con o sin agorafobia (AG). Los resultados que expondremos serán representativos de dos modelos distintos de evaluación y estudio de la personalidad, el modelo de personalidad clínico y el modelo de personalidad normal. Desde ambas perspectivas, parece existir suficiente evidencia para asociar las dimensiones y rasgos patológicos de personalidad más relacionados con la emocionabilidad negativa y la desregulación emocional con el TP, con o sin AG. Entre las variables de vulnerabilidad identificadas en relación a la mayor probabilidad de inicio y mantenimiento del TP destacan: patrones familiares específicos de educación y crianza, la inhibición conductual, la sensibilididad a la ansiedad, y, más recientemente, la percepción de control de la ansiedad. Desde una perspectiva clínica es muy relevante la identificación de estos factores de vulnerabilidad psicopatológica puesto que abren la puerta al diseño e implementación de programas de promoción, prevención y tratamiento que se dirijan específicamente a los procesos psicológicos alterados y mitiguen, con mayor rapidez y eficacia, el sufrimiento de las personas que padecen estas patologías. Palabras clave: Personalidad; trastorno de pánico; revisión; agorafobia; trastornos de la personalidad; factores de riesgo.
\end{abstract}

Title: Personality and panic disorder: a review.

Abstract: In this work we offer a comprehensive review of the scientific contributions that have been made in the last three decades on the influence of personality in the psychopathology of panic disorder (PD) with or without agoraphobia (AG). The findings that are discussed are representative of two different theoretical models in the assessment and the study of personality, the clinical approach to personality and the dimensional approach to personality. From both perspectives, there is sufficient evidence to associate the personality factors and the pathological personality traits more related to negative emotionality and emotional dysregulation with PD, with or without AG. Among the vulnerability factors identified in relation to the onset of PD and the maintenance of its symptoms are: specific family and education patterns, behavioral inhibition, anxiety sensitivity, and, more recently, the perception of anxiety control. From a clinical perspective it is very important to identify these psychopathological vulnerabilities in order to design and implement promotion, prevention and treatment programs that specifically target the disordered psychological processes and reduce, more quickly and effectively, the suffering of people with these conditions.

Key words: Personality; panic disorder; review; agoraphobia; personality disorders; risk factors.

\section{Introducción}

El TP constituye uno de los trastornos mentales que más atención ha recibido por parte de los investigadores (White y Barlow, 2002). De entre los estudios dirigidos al conocimiento de características clínicas y psicopatológicas del TP, en este trabajo abordaremos los relacionados con la influencia de la personalidad.

La aproximación al estudio de la personalidad en el TP, con o sin AG, se ha realizado, principalmente, siguiendo dos métodos tradicionales de evaluación, el primero a través de medidas provenientes de un modelo clínico (Barzega, Maina, Venturello y Bogetto, 2001; Chambles, Renneberg y Goldstein, 1992; Iketani et al., 2002; Marchesi et al., 2006; Telch, Kamphuis y Schmidt, 2011) y el segundo, a través de medidas de un modelo de personalidad normal (Bienvenu et al., 2001, 2004; Bienvenu, Hettema, Neale, Prescott y Kendler, 2007; Carrera et al., 2006; Foot y Koszycki, 2004; Karsten et al., 2012; Kotov, Gamez, Schmidt y Watson, 2010). Los modelos clínicos consideran distintos grupos de signos y síntomas para identificar un trastorno de la personalidad (TPe), requiriendo la presencia de alguno de estos grupos para diagnosticarlo. Por su parte, los modelos de personalidad normal sitúan al sujeto en un continuo que permite definir la presencia de un trastorno en función de la intensidad, fre-

* Dirección para correspondencia [Correspondence address]: Jorge Osma. Universidad de Zaragoza. Facultad de Ciencias Sociales y Humanas de Teruel. Departamento de Psicología y Sociología. Ciudad Escolar s/n, 44003 Teruel (España). E-mail: osma@,unizar.es cuencia o duración de determinados rasgos de personalidad compartidos con la población normal.

La relación existente entre pánico y personalidad deja entrever, por una parte, la posibilidad de que la personalidad (Johnson, Cohen, Brown, Smailes y Bernstein, 1999; Ozkan y Altindag, 2005), u otras variables como situaciones adversas en la infancia (Aronson y Logue, 1987; Brown, Harris y Eales, 1996; Pollack, Otto, Rosenbaum y Sachs, 1992), puedan constituir factores de vulnerabilidad para el desarrollo y curso del TP y, por otra parte, que sea la ansiedad el factor de vulnerabilidad que predispone a la persona a desarrollar no sólo un TP sino también un TPe (Kasen, et al., 2001; Koenigsberg, Kaplan, Gilmore y Cooper, 1985; Lewinsohn, Zinbarg, Seeley, Lewinsohn y Sack, 1997). Estas son las cuestiones que los investigadores se plantean y para las que, a día de hoy, no existen respuestas concluyentes aunque sí una serie de factores de vulnerabilidad o riesgo identificados como posibles candidatos desde perspectivas biológicas, cognitivas y psicosociales.

Desde enfoques biológicos, los factores identificados más estudiados hasta el momento han sido la Sensibilidad a la Ansiedad (Brown, Smits, Powers y Telch, 2003; Reiss, Peterson, Gursky y McNally, 1986; Plehn y Peterson, 2002; Rapee, Brown, Anthony y Barlow, 1992; Weems, Hayward, Killen y Taylor, 2002) y la Inhibición Conductual (Rosenbaum et al., 2000; Segui et al., 1999). Más recientemente, desde enfoques cognitivos, se investiga la posible influencia de la Percepción de Control de la Ansiedad como factor de riesgo en el TP (Baker, Holloway, Thomas, Thomas y Owens, 2004; Barlow, 2002; White y Barlow, 2002). Entre los factores psi- 
cosociales destacamos los patrones familiares de educación y crianza excesivamente rígidos y sobreprotectores (Gittelman y Klein, 1984; Klein, 1964; Latas, Starcevic, Trajkovic y Bogojevic, 2000; Shafar, 1976; Webster, 1953).

Las aportaciones realizadas en las tres últimas décadas sobre la influencia de la personalidad, tanto desde enfoques clínicos como de personalidad normal, en el estudio del TP, con o sin AG, ponen de manifiesto que, la identificación de rasgos de personalidad puede utilizarse para, por una parte, guiar los esfuerzos en prevención e identificar personas con vulnerabilidad para el desarrollo de esta psicopatología y, por otra, realizar mejoras en los programas de tratamiento cognitivo-comportamentales actuales alcanzando mayor eficacia, eficiencia y efectividad.

\section{Método}

\section{Materiales, procedimiento y análisis de la informa- ción}

El presente trabajo es fruto de la tesis doctoral titulada "Influencia de la personalidad en la clínica del trastorno de pánico, con o sin AG: Un estudio exploratorio" (Osma, 2009). Los materiales utilizados para la realización del estudio fueron los artículos científicos, los libros y los manuales cuyo interés se centra en el estudio de la personalidad en muestras de pacientes con TP y trastorno de pánico con agorafobia (TPA). El procedimiento llevado a cabo se ha centrado en la búsqueda bibliográfica de artículos científicos en bases de datos especializadas, así como la búsqueda de libros y manuales sobre la temática de estudio. Posteriormente, se realizó una selección de trabajos en función de los resúmenes, seleccionando aquellos relacionados con las muestras de estudio TP y TPA y aquellos que se relacionaban con la personalidad, bien desde un enfoque de personalidad normal o patológica. Así mismo, para el análisis final de la información se excluyeron los estudios que no utilizaron criterios diagnósticos basados en los Manuales Diagnósticos y Estadísticos de los Trastornos Mentales (DSM) o de la Clasificación Internacional de Enfermedades (CIE), en cualquiera de sus versiones, los que utilizaban muestras de niños y/o adolescentes con TP/TPA y, finalmente, aquellos trabajos que no especificaban el número de participantes con diagnóstico de TP y/o TPA en sus muestras. En el caso concreto de este trabajo, además de seleccionar la información de la tesis doctoral relacionada con la influencia de la personalidad en el TP/TPA, se realizó adicionalmente una actualización de la búsqueda bibliográfica hasta el año 2013.

\section{Bases de datos consultadas y descriptores de bús- queda utilizados}

Para realizar la revisión sistemática de estudios sobre el TP/TPA y personalidad fueron utilizadas dos bases de datos: PsychINFO y PubMed. El período seleccionado de búsqueda fue desde 1985 a 2013. En la primera fase de la búsqueda se utilizaron los siguientes descriptores generales: panic disorder, panic disorder with agoraphobia, panic disorder review y "panic disorder" OR "panic disorder with agoraphobia" AND "meta-analysis". Los descriptores utilizados para obtener información desde enfoques clínicos o psicopatológicos de la personalidad fueron: "personality disorders comorbidity" AND "panic disorder" OR "panic disorder with agoraphobia", panic disorder prevalence y "panic disorder" AND "personality clusters". Para conocer los resultados obtenidos desde enfoques de personalidad normal se utilizaron los siguientes descriptores: " $p a$ nic disorder" OR "panic disorder with agoraphobia" AND "personality dimensions" OR "personality traits" OR "normal personality traits" OR "five-factor" OR "big five". Finalmente, para obtener información sobre los factores de riesgo relacionados con el desarrollo del TP y TPA se utilizó el siguiente descriptor: "panic disorder" OR "panic disorder with agoraphobia" AND" risk factor".

\section{Resultados}

\section{Trastorno de pánico, con o sin agorafobia, y su co- morbilidad con los trastornos de la personalidad}

Alrededor del 70\% de pacientes con TP sufre al menos un trastorno psiquiátrico más (Brown y Barlow, 1992). Además de la comorbilidad con el Eje I, muchos pacientes con TP muestran psicopatología en el Eje II. Los estudios basados en la evidencia empírica utilizan entrevistas clínicas validadas siguiendo los criterios de los Manuales diagnósticos de los trastornos mentales (DSM-IV-TR y CIE-10). Los estudios que utilizan estos criterios de investigación han dado apoyo a la comorbilidad entre algunos TPe y el TP/TPA. El porcentaje de prevalencia encontrado ha ido variando a lo largo de los años, en la década de los 80 y principios de los 90 los trabajos ofrecían porcentajes entre el 40 y el $65 \%$ (Brooks, Baltasar, McDowell, Munjack y Bruns, 1988; Brown y Barlow; 1992; Chambless y Renneberg, 1988; Friedman, Shear y Frances; 1987; Reich, 1988). Desde mitad de los 90 y a principios del 2000 se amplía el porcentaje de prevalencia de los TPe en pacientes con TP/TPA del 20 al 95\% (Ampollini et al., 1999; Barzega, et al., 2001; Berger et al. 2004; Langs et al., 1998; Massion et al. 2002). Los estudios más recientes arrojan cifras que oscilan entre el 33.3 y el $76.8 \%$ (Albert, Maina, Bergesio y Bogetto, 2006; Marchesi, Cantón, Fonito, Giannelli y Maggini, 2005; Marchesi et al., 2006; Ozkan y Altindag, 2005). Como podemos observar existe unanimidad en relacionar los TPe con el TP. Sin embargo, es importante señalar que se produce una gran variabilidad en las estimaciones de la prevalencia. Esta variabilidad es probablemente debida a las diferencias en los instrumentos de evaluación y diagnóstico utilizados y a la población estudiada (Slaap y den Boer, 2001).

Para ofrecer una visión más clarificadora de este apartado hemos realizado una revisión de los trabajos que han evaluado la presencia de TPe en muestras de pacientes con TP/TPA desde el año 1985 hasta el 2013. En la Tabla 1 apa- 
recen los resultados obtenidos por los distintos estudios y, como puede observarse, se pone de manifiesto la relación existente entre el Cluster C (ansioso) de los TPe y el TP/TPA, siendo este Cluster el que obtiene una mayor presencia entre las personas con TP/TPA estudiadas (44.2\%, con un rango de entre un $19 \%$ a un $87 \%$ ). Al Cluster $\mathrm{C}$ le siguen el Cluster B (27.2\%) y el A $(15.7 \%)$. El TPe que obtiene mayor número de casos en el Cluster $\mathrm{C}$, tanto para la muestra conjunta como para la de TP, es el TPe Obsesivocompulsivo $(24.3 \%$ y $29.7 \%$ respectivamente) seguido del TPe Evitativo $(22.7 \%)$ en la muestra conjunta y del TPe Paranoide $(22.3 \%)$ en la muestra con TP. Si tenemos en cuenta las muestras de TPA, obtiene mayor número de casos el TPe Evitativo (27.5\%) seguido del TPe Dependiente (23.9\%).
Como podemos observar, y a pesar de las limitaciones de los estudios en cuanto a las muestras de pacientes y la utilización de distintas y variadas medidas de evaluación y diagnóstico, existe evidencia con respecto a la comorbilidad existente entre el TP/TPA y los TPe. A este respecto destacamos que distintos estudios informan de que los pacientes con TPA parecen mostrar porcentajes más elevados de TPe comparados con los pacientes con TP (Iketani et al., 2002), concretamente, en TPe Evitativo (Alnaes y Torgersen, 1988a, 1988b; Iketani et al., 2002). Este resultado coincide con el obtenido en nuestro estudio de revisión (Tabla 1). También se ha informado que los pacientes con TP y puntuaciones elevadas en evitación fóbica experimentan mayores porcentajes de TPe (Friedman et al., 1987).

Tabla 1. Porcentajes de diagnósticos de TPe específicos en muestras de TP y TPA descritos en la literatura incluyendo las medidas diagnósticas utilizadas.

\begin{tabular}{|c|c|c|c|c|c|c|c|c|c|c|c|c|c|c|c|}
\hline Autores & Medidas & Muestra & $\begin{array}{l}\mathbf{P a} \\
\%(\mathrm{~N})\end{array}$ & $\begin{array}{l}\text { Es } \\
\%(N)\end{array}$ & $\begin{array}{l}\text { Esq } \\
\%(\mathrm{~N})\end{array}$ & $\begin{array}{l}\text { Cluster } \\
\text { A* }^{*} \%(\mathrm{~N}) \\
\end{array}$ & $\begin{array}{l}\mathbf{N a} \\
\%(\mathrm{~N}) \\
\end{array}$ & $\begin{array}{l}\mathbf{H i} \\
\%(\mathrm{~N})\end{array}$ & $\begin{array}{l}\text { Lí } \\
\%(\mathrm{~N})\end{array}$ & $\begin{array}{l}\text { An } \\
\%(N)\end{array}$ & $\begin{array}{l}\text { Cluster } \\
\text { B* } \\
\%(\mathrm{~N}) \\
\end{array}$ & $\begin{array}{l}\text { Ev } \\
\%(N)\end{array}$ & $\begin{array}{l}\text { De } \\
\%(N)\end{array}$ & $\begin{array}{l}\text { Ob } \\
\%(\mathrm{~N})\end{array}$ & $\begin{array}{l}\text { Cluster } \\
\mathrm{C}^{*} \\
\%(\mathrm{~N}) \\
\end{array}$ \\
\hline $\begin{array}{l}\text { Mavissakalian y } \\
\text { Hamann, } 1986\end{array}$ & $\begin{array}{l}\text { HSCL, PDQ y } \\
\text { EPI }\end{array}$ & TP:60 & - & - & - & - & - & - & - & - & - & $38(23)$ & $15(9)$ & - & - \\
\hline $\begin{array}{l}\text { Mavissakalian y } \\
\text { Hamann, } 1987\end{array}$ & PDQ & TP:33 & - & - & - & - & - & $15(5)$ & - & - & - & $15(5)$ & $12(4)$ & - & - \\
\hline Brooks et al., 1988 & $\begin{array}{l}\text { SCID-UP, } \\
\text { SCID-II, } \\
\text { MCMI }\end{array}$ & TP:158 & - & - & - & - & - & - & - & - & - & $22(35)$ & $4(6)$ & - & - \\
\hline $\begin{array}{l}\text { Chambless y Ren- } \\
\text { neberg, } 1988\end{array}$ & $\begin{array}{l}\text { SCID-II, } \\
\text { MCMI-I y II }\end{array}$ & TP:20 & - & - & - & - & - & - & - & - & - & $33(7)$ & - & - & - \\
\hline Green y Curtis, 1988 & 8SCID-II & TP:19 & - & - & - & - & - & - & - & - & - & $20(4)$ & $8(2)$ & - & - \\
\hline Reich, 1988 & $\begin{array}{l}\text { SCID, SIDP, } \\
\text { PDQ, MCMI }\end{array}$ & TP:52 & - & - & - & - & - & - & - & - & - & $19(10)$ & $25(13)$ & - & - \\
\hline $\begin{array}{l}\text { Mavissakalian y } \\
\text { Hamman, } 1988\end{array}$ & PDQ & $\begin{array}{l}\text { TPA:161 } \\
\text { TP:26 }\end{array}$ & - & - & $9(17)$ & - & - & $13(24)$ & $2(4)$ & - & - & $21(39)$ & $19(35)$ & - & - \\
\hline $\begin{array}{l}\text { Reich y Troughton, } \\
1988\end{array}$ & SIDP & $\begin{array}{l}\text { TPA:37 } \\
\text { TP:51 }\end{array}$ & - & - & $0(0)$ & - & - & $10.2(9)$ & $6.8(6)$ & - & - & $20.5(18)$ & $18.2(16)$ & - & - \\
\hline $\begin{array}{l}\text { Reich y Troughton, I } \\
1988\end{array}$ & PDQ & $\begin{array}{l}\text { TPA:37 } \\
\text { TP:51 }\end{array}$ & - & - & )$^{34.2(30}$ & & - & $17(15)$ & $15.9(14)$ & - & - & $13.4(12)$ & $26.8(24)$ & - & - \\
\hline $\begin{array}{l}\text { Alnaes y Torgersen, } \\
1990\end{array}$ & $\begin{array}{l}\text { SCID-I, } \\
\text { MCMI-I }\end{array}$ & TP:38 & $11(4)$ & $29(11)$ & $18(7)$ & - & $5(2)$ & $29(11)$ & $40(15)$ & $8(3)$ & - & $40(15)$ & $45(17)$ & $5(2)$ & - \\
\hline $\begin{array}{l}\text { Chambles, Renne- } \\
\text { berg y Goldstein, } \\
1992\end{array}$ & $\begin{array}{l}\text { SCID-I, } \\
\text { MCMI-I }\end{array}$ & TPA:48 & $4(2)$ & $25(12)$ & $10(5)$ & $30(14)$ & $6(3)$ & $35(17)$ & $48(23)$ & $0(0)$ & $68(33)$ & $38(18)$ & $67(32)$ & $4(2)$ & $87(42)$ \\
\hline $\begin{array}{l}\text { Chambles, Renne- } \\
\text { berg y Goldstein, } \\
1992\end{array}$ & $\begin{array}{l}\text { SCID-I, } \\
\text { MCMI-II }\end{array}$ & TPA:117 & $7(8)$ & $24(28)$ & $10(12)$ & $31(36)$ & $21(25)$ & $31(36)$ & $18(21)$ & $15(17)$ & $49(57)$ & $39(46)$ & $44(51)$ & $25(29)$ & $79(92)$ \\
\hline $\begin{array}{l}\text { Chambles, Renne- } \\
\text { berg y Goldstein, } \\
1992\end{array}$ & MCMI-I & TPA:64 & - & - & - & $25(16)$ & - & - & - & - & $36(23)$ & - & - & - & $80(51)$ \\
\hline $\begin{array}{l}\text { Reich y Braginsky, } \\
1994\end{array}$ & PDQ-R & $\begin{array}{l}\text { TPA:23 } \\
\text { TP:5 }\end{array}$ & $54(15)$ & - & - & $64(18)$ & - & $25(7)$ & - & - & $39(11)$ & $46(13)$ & $36(10)$ & $25(7)$ & $64(18)$ \\
\hline $\begin{array}{l}\text { Hoffart y Hedley, } \\
1997\end{array}$ & $\begin{array}{l}\text { SCID-I, SCID- } \\
\text { II }\end{array}$ & -TPA:46 & $6.5(3)$ & - & - & - & - & - & - & - & - & $50(23)$ & $17.4(8)$ & $13(6)$ & - \\
\hline Langs et al., 1998 & SCID-I y II & TP:84 & - & - & - & - & $13(11)$ & $12(10)$ & - & - & - & - & $17(14)$ & $17(14)$ & - \\
\hline $\begin{array}{l}\text { Marchand, Giles, } \\
\text { Dupuis y Mainguy, } \\
1998\end{array}$ & $\begin{array}{l}\text { Criterios DSM- } \\
\text { III-R }\end{array}$ & -TPA:81 & - & - & - & - & $1.2(1)$ & $6.2(5)$ & - & - & - & $4.9(4)$ & $22.2(18)$ & $2.5(2)$ & - \\
\hline Starcevic et al., $1999 \mathrm{~s}$ & SCID-I y II & TPA: 88 & $19.3(17)$ & $3.4(3)$ & $0(0)$ & $20.45(18)$ & $10.2(9)$ & $13.6(12)$ & $22.7(20)$ & 1.1(1) & $27.3(24)$ & $14.8(13)$ & $22.7(20)$ & $17(15)$ & $39.8(35)$ \\
\hline Latas et al., 2000 & SCID-II & TPA: 60 & $15(9)$ & - & - & $17(10)$ & - & $10(6)$ & $20(12)$ & - & $25(15)$ & $12(7)$ & $15(9)$ & 18(11) & $28(17)$ \\
\hline Barzega et al., 2001 & $\begin{array}{l}\text { SCID-I, SCID- } \\
\text { PQ, MCMI, } \\
\text { PDQ }\end{array}$ & - TP:184 & $1.6(3)$ & $6.5(12)$ & $0.5(1)$ & $6.5(12)$ & $3.8(7)$ & $9.8(18)$ & $13.6(25)$ & $0(0)$ & $25(46)$ & $21.7(40)$ & $26.6(49)$ & $15.8(29)$ & $45.6(84)$ \\
\hline Iketani et al., 2002 & $\begin{array}{l}\text { SCID-II, } \\
\text { FMPS, SCID-P }\end{array}$ & TPA:56 & $19.6(11)$ & - & - & - & - & - & - & - & - & $35.7(20)$ & $25(14)$ & $30.3(17)$ & - \\
\hline Iketani et al., 2002 & $\begin{array}{l}\text { SCID-II, } \\
\text { FMPS, SCID-P }\end{array}$ & $\mathrm{P}^{\mathrm{TP}: 42}$ & - & - & - & - & $16.7(7)$ & - & $14.3(6)$ & - & - & $16.7(7)$ & $14.3(6)$ & - & - \\
\hline Massion et al., 2002 I & ICD-10 & TPA:313 & - & 1(3) & $0(0)$ & - & 1(3) & $2(6)$ & $4(12)$ & - & - & $12(38)$ & $5(16)$ & $5(16)$ & - \\
\hline Massion et al., $2002 \mathrm{I}$ & ICD-10 & TP:73 & - & $0(0)$ & $1(1)$ & - & $0(0)$ & $3(2)$ & $3(2)$ & - & - & $14(10)$ & $3(2)$ & $5(4)$ & - \\
\hline $\begin{array}{l}\text { Venturello, Barzega, } \\
\text { Maina y Bogetto, } \\
2002\end{array}$ & $\begin{array}{l}\text {, SCID-I, SCID- } \\
\text { PQ }\end{array}$ & - TP:241 & - & - & - & $15.3(37)$ & - & - & - & - & $27.8(67)$ & $17.8(43)$ & $24(58)$ & $19.9(48)$ & $45.2(109)$ \\
\hline Iketani et al., 2004 & $\begin{array}{l}\text { SCID-P, } \\
\text { SCID-II }\end{array}$ & TP:105 & $12.4(13)$ & $2.8(3)$ & $1.9(2)$ & $13.3(14)$ & $14.3(15)$ & $6.7(7)$ & $13.3(14)$ & $8.6(9)$ & $31.4(33)$ & $22.8(24)$ & 17.1(18) & $19(20)$ & $44.8(47)$ \\
\hline
\end{tabular}




\begin{tabular}{|c|c|c|c|c|c|c|c|c|c|c|c|c|c|c|c|}
\hline Grant et al., 2005 & AUDADIS-IV & TPA:259 & $35.5(92)$ & $28.6(74)$ & - & - & - & $12.8(33)$ & - & $17.3(45)$ & - & $32(83)$ & $13.5(35)$ & $38.1(99)$ & - \\
\hline Grant et al., 2005 & AUDADIS-IV & $\mathrm{TP}: 690$ & $19.6(135)$ & $11.4(79)$ & - & - & - & $7.2(50)$ & - & $12.1(83)$ & - & $10.9(75)$ & $2.7(19)$ & $23.7(163)$ & - \\
\hline $\begin{array}{l}\text { Ozkan y Altindag, } \\
2005\end{array}$ & SCID-I y II & $\begin{array}{l}\text { TPA:73 } \\
\text { TP:39 }\end{array}$ & - & - & - & $7.1(8)$ & $7.1(8)$ & $15.2(17)$ & $14.3(16)$ & - & $23.2(26)$ & $8.9(10)$ & $7.1(8)$ & $11.6(13)$ & $25(28)$ \\
\hline Marchesi et al, 2005 & SCID-I, SIDP & TP:60 & $11.6(7)$ & $1.6(1)$ & $0(0)$ & $11.6(7)$ & $13.3(8)$ & $5(3)$ & $5(3)$ & $0(0)$ & $21.6(7)$ & $11.6(7)$ & $13.3(8)$ & $18.3(11)$ & $40(24)$ \\
\hline Marchesi et al., 2006 & 6 SCID-I, SIDP & TP:71 & $9.9(7)$ & 1.4(1) & $1.4(1)$ & $9.9(7)$ & $12.7(9)$ & $4.2(3)$ & $5.6(4)$ & $0(0)$ & 19.7(14) & $11.3(8)$ & $12.7(9)$ & $18.3(13)$ & $36.6(26)$ \\
\hline Albert et al., 2006 & SCID-I y II & TP:116 & $0.9(1)$ & $6(7)$ & $2.6(3)$ & $7.8(9)$ & $3.4(4)$ & $7.8(9)$ & $7.8(9)$ & $0(0)$ & $19(22)$ & $9.5(11)$ & $15.5(18)$ & $11.2(13)$ & $32.8(38)$ \\
\hline $\begin{array}{l}\text { Milrod, Leon, Bar- } \\
\text { ber, Markowitz y } \\
\text { Graf, } 2007\end{array}$ & $\begin{array}{l}\text { Criterios DSM- } \\
\text { IV, SCID-II }\end{array}$ & -TP:49 & - & - & - & - & - & - & - & - & - & - & - & - & $39(19)$ \\
\hline $\begin{array}{l}\text { Starcevic, et al., } \\
2008\end{array}$ & SCID-I y II & TPA:157 & 19.1(30) & $3.2(5)$ & $0(0)$ & $21(33)$ & $8.9(14)$ & $10.2(16)$ & $8.3(13)$ & $1.9(3)$ & $21.7(34)$ & $14.6(23)$ & $22.9(36)$ & $9.6(15)$ & $38.9(61)$ \\
\hline $\begin{array}{l}\text { Mendoza, et al., } \\
2011\end{array}$ & $\begin{array}{l}\text { SCID-I y II, } \\
\text { TCI }\end{array}$ & TP:95 & - & - & - & $1(1)$ & - & - & - & - & $9.5(9)$ & - & - & - & $19(18)$ \\
\hline $\begin{array}{l}\text { Telch, Kamphuis y } \\
\text { Schmidt, } 2011\end{array}$ & SCID-II & TP:173 & $5.2(9)$ & $0(0)$ & $1.7(3)$ & $6.9(12)$ & $1.7(3)$ & $1.7(3)$ & $5.8(10)$ & $1.2(2)$ & $8.7(15)$ & $21.4(37)$ & $6.9(12)$ & $5.8(10)$ & $24.3(42)$ \\
\hline Media de casos TP & & & 21.5 & 15.9 & 5.1 & 15.7 & 7.6 & 13.5 & 12.1 & 12.5 & 27.2 & 22.7 & 18.6 & 24.3 & 44.2 \\
\hline y TPA (36 estu- & & & 22.3 & 12.7 & 0.4 & 12.4 & 6.6 & 11.0 & 9.8 & 12.1 & 26.6 & 21.2 & 16.6 & 29.7 & 45.2 \\
\hline dios) & & & 21.5 & 20.8 & 3.4 & 21.2 & 9.2 & 16.5 & 16.8 & 13.2 & 31 & 27.5 & 23.9 & 21.2 & 49.7 \\
\hline $\begin{array}{l}\text { Media de casos TP } \\
\text { ( } 20 \text { estudios) } \\
\text { Media de casos } \\
\text { TPA (11 estudios) }\end{array}$ & & & & & & & & & & & & & & & \\
\hline
\end{tabular}

TPA (11 estudios)

Nota: TPe: Trastorno de la personalidad; TP: Trastorno de pánico; TPA: Trastorno de pánico con agorafobia; Pa: Paranoide; Es: Esquizoide; Esq: Esquizotípico; Na: Narcisista; Hi: Histriónico; Li: Límite; An: Antisocial; Ev: Evitativo; De: Dependiente; Ob: Obsesivo; HSCL: Hopkins Symptom Checklist; PDQ: Personality Disorder Questionnaire; EPI: Eysenk Personality Inventory; SCID: Structured Clinical Interview for DSM Disorders; SCID-PQ: Computerassisted Structured Clinical Interview II Personality Questionnaire; MCMI: Inventario Clínico Multiaxial de Millon; SIDP: Structured Interview for DSM-IV Personality; DSM: Manual Diagnóstico y Estadístico de los Trastornos Mentales; FMPS: Multidimensional Perfectionism Scale; ICD: International Classification of Diseases; AUDADIS: Alcohol Use Disorders and Associated Disabilities Interview Shedule; TCI: Temperament and Character Inventory.

\section{Aportaciones de la investigación basada en un enfo- que de personalidad clínica o patológica en mues- tras con trastorno de pánico, con o sin agorafobia}

La comparación de características de personalidad entre grupos de pacientes con TP/TPA y grupos control, nos ha ofrecido un mayor conocimiento sobre las diferencias existentes entre los rasgos de personalidad "normales" y "patológicos" (según las medidas basadas en modelos clínicos) y también, las combinaciones de dimensiones y facetas de personalidad que más se asocian con un trastorno mental específico (medidas basadas en modelos de personalidad normal). Existen estudios cuyo interés se ha centrado en delimitar si existen perfiles específicos entre subgrupos de pánico, es decir, si las características de personalidad son diferentes en pacientes con TP de los que tienen TPA o AG sin historia de pánico, o si son similares estas características entre pacientes con inicio temprano del problema de aquellos con inicio tardío, entre adolescentes y adultos, y hombres y mujeres, entre otros. Veamos los resultados obtenidos desde el modelo de personalidad clínico.

Los modelos clínicos o psicopatológicos de la personalidad, además de los hallazgos obtenidos respecto a la comorbilidad con Eje II en el TP/TPA, han obtenido resultados interesantes respecto a la comorbilidad con Eje I. Algunos autores han puesto de manifiesto que la sintomatología depresiva se relaciona con el aumento de la probabilidad de tener un TPe (Alnaes y Torgersen, 1990; Chambless et al., 1992; Klass, DiNardo y Barlow, 1989; Ozkan y Altindag, 2005; Reich y Troughton, 1988; Reich et al., 1994) y también que los pacientes con trastornos de ansiedad y comorbilidad con Eje II obtienen mayores porcentajes de síntomas depresivos (Klass et al., 1989). A este respecto, cabe mencionar los estudios que relacionan el TP con la ideación o intentos suicidas. Los estudios indican que la ideación suicida se relaciona con síntomas depresivos, adicción a sustancias y comorbilidad en Eje II (Lepine, Chignon y Teherani, 1993) o también con presencia de depresión, TPe y mayor gravedad del TPA (Starcevic et al., 1999). Los pacientes con TP y comorbilidad en Eje II manifiestan sintomatología más grave y peores puntuaciones en funcionamiento social (Chambless et al., 1992; Ozkan y Altindag, 2005; Segui et al., 1999; Seivewright, Tyrer y Johnson, 2004; Reich et al., 1994). Se ha encontrado también una asociación entre la mayor incidencia de TPe y abuso de sustancias, ansiedad y trastornos somatoformes (Koenigsberg et al., 1985).

Se han realizado estudios que relacionan las variables clínicas y el diagnóstico de TPe con la edad de inicio del TP. Los pacientes con inicio temprano (menos de 18 ó 25 años) presentaban mayor frecuencia de antecedentes familiares de trastornos psicológicos, mayor frecuencia de historia de trastornos de ansiedad (ansiedad por separación), dismorfofobia y bulimia nerviosa, mayor comorbilidad con TPe, concretamente el Cluster $\mathrm{C}$, ocurrencia de al menos un diagnóstico más en Eje I y mayores niveles de ansiedad y depresión (Venturello et al., 2002). También se asocia el inicio temprano con una mayor gravedad de los síntomas y un aumento de la prevalencia de la AG (Battaglia et al., 1997; Iketani et al., 2004; Ozkan y Altindag, 2005).

Los estudios que han tenido en cuenta las diferencias en función del sexo en la génesis, clínica y curso del TP/TPA son más numerosos que los que han estudiado las diferencias en TPe en función del sexo en pacientes diagnosticados con TP/TPA. Según estos estudios los hombres obtienen mayores porcentajes de diagnóstico en cluster A, especialmente, en TPe Paranoide y Esquizoide (Reich, 1987; Reich et al., 1994; Barzega et al., 2001). El TPe más frecuente en hombres es el TPe Evitativo y Compulsivo (Reich, 1987; 
Reich y Troughton, 1987) y el TPe Histriónico es el más diagnosticado en mujeres de los TPe del cluster B (Barzega et al., 2001).

\section{Aportaciones de la investigación basada en un enfo- que de personalidad normal en muestras con tras- torno de pánico, con o sin agorafobia}

Desde modelos de personalidad normal se pone de manifiesto que las personas con trastornos de ansiedad tienden a ser perfeccionistas (Antony, Purdon, Huta y Swinson, 1998; Frost y Steketee, 1997; Matsunaga et al., 2000; Uhlenhuth et al., 2002), este dato se confirma también en el caso concreto de los pacientes con TP (Antony et al., 1998) y en mayor porcentaje en pacientes con TPA cuando existe comorbilidad con TPe (Iketani et al., 2002). Según Iketani y colaboradores (2002) los indicadores más robustos de perfeccionismo son la comorbilidad con el Cluster C, concretamente con TPe Evitativo y Obsesivo-compulsivo.

La dimensión de personalidad más relacionada con los trastornos de ansiedad y los trastornos del estado de ánimo ha sido el Neuroticismo (Bienvenu et al., 2001, 2004; Bramsen, Dirkzwager y van der Ploeg, 2000; Fauerbach, Lawrence, Schmidt, Munster y Costa, 2000; Jankowsky, Morter y Tancer, 2000; Kotov et al., 2010; Samuels et al., 2000). Puntuaciones elevadas en Neuroticismo se asocia con: aumento del estrés negativo asociado a situaciones vitales (Ormel y Wohlfarth, 1991; Poulton y Andrews, 1992), aislamiento social, autodescripciones relacionadas con preocupaciones excesivas, ansiedad, sentimientos de victimización y resentimiento (Costa y McCrae, 1980; Eysenk, 1982; Watson y Pennebaker, 1989).

Otros estudios encuentran en sus muestras de TP junto al elevado Neuroticismo, la baja Extraversión, y las relacionan con mayor gravedad y generalización de los síntomas y mayor número de eventos indeseables un año antes de la evaluación (Bienvenu et al., 2004; Carrera et al., 2006; Mavissakalian y Hamann, 1988), además de una mayor incapacidad en el funcionamiento y mayor comorbilidad con Eje I (Katon et al., 1995). Klass y sus colaboradores (1989) encontraron en su estudio mayor correlación entre las variables: Neuroticismo, estrés, estado de ánimo disfórico, ansiedad social y duración y gravedad de TPA.

Junto con los estudios que relacionan factores de primer orden con TP/TPA o AG, otros estudios se interesan por encontrar mayor especificidad entre trastornos por lo que buscan diferencias en las facetas de personalidad de segundo orden. En el trabajo de Bienvenu y colaboradores (2001), se concluye que los pacientes con AG obtienen puntuaciones altas en la mayoría de facetas del Neuroticismo y bajas en Extraversión. Obtienen bajas puntuaciones en las facetas confianza y actitud conciliadora del factor Amabilidad y baja autodisciplina en el factor Responsabilidad. Los pacientes del grupo TP obtienen altas puntuaciones en todas las facetas del Neuroticismo y bajas en las facetas: emociones positivas, cordialidad y asertividad del factor Extraversión; tam- bién presentan puntuaciones bajas en las facetas confianza y actitud conciliadora del factor Amabilidad y bajas en la faceta competencia del factor Responsabilidad. En su trabajo de 2004, Bienvenu y colaboradores, encuentran de nuevo perfiles distintos entre pacientes con TP y AG. Aunque ambos puntuaban alto en Neuroticismo y bajo en emociones positivas, los pacientes con AG puntuaban bajo en Extraversión, sobre todo en la faceta de cordialidad y también bajo en la faceta confianza de la dimensión Amabilidad. Los pacientes con TP también puntuaban bajo en autodisciplina, faceta de la Responsabilidad.

Respecto a las diferencias en función del sexo, el estudio de Foot y Koszycki (2004) informa que tanto hombres como mujeres obtienen altas puntuaciones en Neuroticismo aunque destacan que los hombres puntúan más alto en las subescalas hostilidad y depresión. Las mujeres puntúan más alto que los hombres en Extraversión. Carrera et al. (2006), destacan que los pacientes eran más introvertidos cuando presentaban AG; otros predictores para la AG eran ser mujer y la gravedad de las crisis.

Finalmente, el trabajo de meta-análisis de Kotov et al. (2010) realizado con 175 estudios publicados desde 1980 a 2007 en muestras con trastornos de ansiedad, del estado de ánimo y trastornos adictivos pone de manifiesto que, todos estos trastornos mentales están fuertemente relacionados con la personalidad, con puntuaciones en el tamaño del efecto superiores a 2.0. Todos los trastornos estudiados, incluyendo el TP y la AG, se definieron por puntuaciones altas en Neuroticismo y bajas en Responsabilidad.

\section{Factores de riesgo asociados al trastorno de pánico, con o sin agorafobia}

Para el desarrollo de estrategias preventivas en psicopatología es crucial el estudio de los factores de riesgo. Existen ciertas cualidades, observables en los primeros estadios de la vida, que predisponen al niño al posterior desarrollo del TP según la investigación en características de personalidad. Además de las variables parentales relacionadas con los estilos educativos y características psicopatológicas de los progenitores, tres rasgos de personalidad han recibido más atención con respecto al TP: la inhibición conductual (IC), la sensibilidad a la ansiedad (SA) y más recientemente, la percepción de control de la ansiedad (PCA). Describiremos a continuación los hallazgos más importantes de estos factores de vulnerabilidad o riesgo.

\section{Variables parentales y ansiedad en la infancia}

Una dificultad en los estudios con familias es determinar qué factores de vulnerabilidad están genética o ambientalmente determinados. No obstante, dado que los estudios sobre heredabilidad de los trastornos de ansiedad obtienen resultados parciales, es comprensible que los factores del ambiente puedan ser también importantes en su génesis. Algunos autores plantean, a través de investigaciones longitu- 
dinales, que aquellos niños/as criados/as en familias con padres sobreprotectores y rígidos obtienen más porcentajes en TPA y TPe en la edad adulta (Gerlsma, Emmelkamp y Arrindell, 1990; Latas et al., 2000; Parker y Gladstone, 1996). Puede que estas circunstancias sumadas a la posibilidad de experimentar eventos estresantes en la infancia y adolescencia (Moisan y Engels, 1995; Pollack et al., 1992), aumenten la posibilidad de desarrollar rasgos de personalidad evitativos y/o dependientes desde la juventud y aumentar la probabilidad del inicio de un TPe Evitativo o Dependiente en la edad adulta, así como el desarrollo y mantenimiento del TP, con o sin AG (Johnson et al., 1999; Kasen et al., 2001; Lewinsohn et al., 1997; Nystrom y Lindergard, 1975). Otros determinantes estudiados que pudieran influir en el desarrollo de rasgos de personalidad y trastornos de ansiedad y depresión han sido: padres con problemas económicos, abuso y pérdida temprana de los padres (Kendler et al., 2000a; Kendler, Meyers y Prescott, 2000b; Reti et al., 2002a; Reti et al., 2002b). Finalmente, otros autores han encontrado que las personas que han sufrido ansiedad por separación en la infancia tienen mayor probabilidad de desarrollar TP y TPe en la edad adulta (Aronson y Logue, 1987; Venturello et al., 2002).

\section{Inbibición Conductual}

Descrita por Kagan y colaboradores (Kagan, Snidman, Arcus y Reznick, 1994), la IC hace referencia a un rasgo del temperamento caracterizado por la tendencia a ser cauto, callado y conductualmente reservado en situaciones no familiares. Una serie de estudios longitudinales realizados en los años 80 demostraron que esta tendencia en situaciones nuevas es un rasgo relativamente estable durante la infancia y hasta la adolescencia (Caspi y Silva, 1995; García-Coll, Kagan, y Reznick, 1984). Segui y sus colaboradores (1999) en su estudio con personas cuyo diagnóstico de TP se produjo en edades muy tempranas concluye que el inicio temprano del TP deriva de un factor patogénico común representado por una dimensión temperamental definida como "inhibición conductual". Este factor puede conducir a una fobia escolar $\mathrm{u}$ otros trastornos de ansiedad de inicio en la infancia y adolescencia y, en un futuro, a trastornos de ansiedad más graves como la fobia social o el TP. Algunos autores han descrito los rasgos de personalidad de estos niños, como por ejemplo: introversión, inhibición social, no participar cuando se les pregunta o ser poco asertivos cuando son adultos, baja autoestima y niveles elevados de sensibilidad interpersonal (Biederman et al., 1993; Rosenbaum, Biederman, Pollack y Hirshfeld, 1994).

La asociación entre IC y TPA se ha observado en estudios comparando hijos de personas que sufren TPA (con o sin comorbilidad con depresión) con los hijos de padres con depresión u otros trastornos psiquiátricos. Estos estudios han demostrado una notable presencia de IC en los hijos de padres con TPA comparados con los hijos de padres sin TPA o trastorno depresivo mayor (Battaglia et al., 1997;
Manassis, Bradley, Goldberg, Hood, y Swinson, 1995). Reich (1991) encontró que existen más rasgos dependientes y evitativos en las primeras generaciones de pacientes con TP comparado con los controles. Otros estudios sugieren que la relación entre el TP en padres y la IC de los hijos está, mayoritariamente causada, por la comorbilidad del TP de los padres con depresión mayor (Rosenbaum et al., 2000). Específicamente, estos autores encontraron una mayor proporción de IC en hijos con padres que tenían TP y depresión mayor, y no encontraron diferencias en los porcentajes entre los hijos de padres con depresión o con TPA.

Utilizando autoinformes retrospectivos sobre IC durante la infancia, Reznick, Hegeman, Kaufman y Woods (1992) encontraron altos porcentajes en pacientes con TP adultos comparados con los adultos controles. Encontraron datos similares con adultos que sufrían depresión. Estudios longitudinales sugieren que la IC estaría relacionada con el consecuente desarrollo de trastornos de ansiedad pero falta especificidad para considerarlo factor de riesgo para el TP (Caspi, Moffitt, Newman y Silva, 1996; Eaton, Kessler, Wittchen y Magee, 1994; Schwartz, Snidman y Kagan, 1999). Futuros estudios longitudinales con niños en riesgo serán necesarios para confirmar sí la IC temprana se relaciona con el posterior inicio del TP (Rosenbaum et al., 1993).

\section{Sensibilidad a la Ansiedad}

La SA hace referencia a la tendencia a responder con temor a las sensaciones relacionadas con la ansiedad (Reiss et al., 1986). McNally (2002) enfatiza que la SA es distinta a la ansiedad rasgo, que denota la tendencia a responder con ansiedad ante los estresores en general en lugar de referirse a un miedo específico a la ansiedad. Weems et al. (2002) estudiaron a 2.365 adolescentes con distintos grados de SA a lo largo de un período de cuatro años y encontraron pequeñas fluctuaciones en SA. Estos resultados se han observado también en muestras de personas adultas por lo que se considera a la SA un rasgo permanente.

Distintas aproximaciones han mostrado evidencias relacionando la SA con el TP. Primero, los estudios descriptivos muestran que los pacientes con TP obtienen puntuaciones más elevadas en el Índice de Sensibilidad a la Ansiedad (ASI; Reiss et al., 1986) comparados con controles psiquiátricos y no psiquiátricos (Taylor, Koch y McNally, 1992; Telch, Jacquin, Smits y Powers, 2003). Segundo, estudios de laboratorio donde se observan los síntomas de ansiedad inducidos artificialmente (hiperventilación voluntaria o inhalación de aire enriquecido con CO2), muestran que los pacientes con TP responden con mayores niveles de ansiedad comparados con controles psiquiátricos y no psiquiátricos (Gorman et al., 1994; Papp et al., 1997; Telch et al., 2003). Además, la SA es un predictor del nivel de respuesta emocional, aquellos que obtienen mayores puntuaciones en el ASI presentan mayores niveles de ansiedad y pánico (Brown, et al., 2003; Rapee et al., 1992). Tercero, el éxito en el tratamiento del TP se asocia con el descenso de las puntuaciones del ASI (Penava, 
Otto, Maki y Pollack, 1998; Simon et al., 2004) y la mejora en la respuesta emocional en los ensayos del laboratorio (Schmidt, Trakowski y Staab, 1997). Evidencias preliminares sugieren que la mejoría producida en los síntomas del TP mediante un tratamiento cognitivo-comportamental está mediada por cambios en el miedo a las sensaciones relacionadas con la ansiedad (Smits, Powers, Cho y Telch, 2004).

Aunque los datos recogidos en estos estudios indican que la SA está implicada en el mantenimiento del TP, no indicarían que la SA es un factor de riesgo para desarrollar TP. Según McNally (2002), la SA puede considerarse como factor de riesgo para desarrollar TP en el caso de que se observe en personas sin historia de pánico. En este sentido, un gran número de estudios han demostrado que la SA puede estar presente sin una experiencia previa de pánico (Asmundson y Norton, 1993). Por ejemplo, Asmundson y Norton (1993) administraron el ASI a una gran muestra de estudiantes de instituto y encontraron que el 70\% de los estudiantes con una alta SA nunca habían experimentado un ataque de pánico (AP) inesperado, y el 45\% nunca habían experimentado un AP situacional.

Entonces, ¿están las personas con alta SA en riesgo de desarrollar TP? Esta pregunta fue formulada en primer lugar por Maller y Reiss (1992) en un estudio de seguimiento de tres años con una muestra de 151 estudiantes de instituto sin historia de AP. Los autores del estudio encontraron que las puntuaciones obtenidas en la línea de base en el ASI predecían la frecuencia e intensidad de los AP en el periodo de seguimiento. Además, los participantes con niveles más elevados de SA obtuvieron una probabilidad cinco veces mayor de desarrollar un trastorno de ansiedad. Schmidt, Lerew y Jackson (1997) evaluaron a 1.172 cadetes de la Academia de las Fuerzas Aéreas Americanas durante las primeras y últimas semanas de su entrenamiento. La SA predijo el desarrollo de AP espontáneos incluso controlando las variables de historia de AP y rasgo de ansiedad. Los AP espontáneos se experimentaron en aproximadamente el $20 \%$ de las personas con altos niveles de SA mientras esto ocurría en el resto de la muestra en un porcentaje de un $6 \%$. Estudios prospectivos (Schmidt, et al., 1997) apoyan la idea de que la SA actúa como un factor de predisposición para el desarrollo del TP. Cox, Enns, Walker, Kjernisted y Pidlubny (2001) intentaron identificar factores de vulnerabilidad en depresión y TP. Basados en el modelo tripartito de Clark (Clark y Watson, 1991) utilizan tanto factores de orden superior (arousal somático y afecto positivo) como de orden inferior (SA, rumiación, autocrítica y perfeccionismo) para determinar qué variables emergen como características en cuanto a la vulnerabilidad a desarrollar un TP. El estudio se realizó con 38 pacientes con diagnóstico de depresión y el mismo número de pacientes con diagnóstico de TP. Los resultados obtenidos indicaron que, con respecto a los factores de orden superior, el Neuroticismo se ha identificado como una variable potente pero relativamente no específica como factor de riesgo para muchas formas de psicopatología, y en este estudio, fracasó en la diferenciación entre pacientes deprimidos y ansiosos. La única variable que consiguió con éxito diferenciar a los pacientes deprimidos de los pacientes con TP fue el arousal y la Extraversión. De las 4 variables estudiadas de orden inferior aparecen como predictoras únicamente la SA (miedo a los síntomas físicos) y el estilo de respuesta basado en la rumiación.

Plehn y Peterson (2002) realizaron un estudio longitudinal que comenzó entre los años 1986 y 1988 y finalizó en 1999. De los 505 individuos que participaron en la primera evaluación la muestra se redujo a 178 y los resultados obtenidos indicaron que tanto la SA como la ansiedad rasgo fueron variables predictoras del desarrollo de síntomas de pánico. Los resultados también indicaron que la SA fue el único predictor significativo de la ocurrencia de AP. Asimismo la historia de pánico y la ansiedad rasgo predijeron la ocurrencia del TP aunque este resultado pudo ser debido al reducido número de diagnósticos de TP, cinco concretamente. Finalmente, con respecto a la predicción de rasgos de ansiedad, la SA no apareció como variable predictora. Los autores concluyeron que identificar la SA como factor de riesgo para el desarrollo de síntomas de pánico y AP es muy relevante para la detección e implementación de intervenciones basadas en modelos cognitivo-comportamentales que reduzcan el riesgo de desarrollar síntomas de ansiedad o un TP.

Como podemos observar, muchos estudios longitudinales han confirmado que las personas que puntúan alto en el ASI tienen mayor riesgo de experimentar AP comparado con aquellos que obtienen puntuaciones más bajas (Cox et al., 2001; Hayward, Killen, Kraemer y Taylor, 2000; Plehn y Peterson 2002; Schmidt, Lerew y Jackson, 1999). Sin embargo, una nota de Schmidt et al. (1999) aclara que la SA únicamente explica una mínima parte de la varianza en la ocurrencia del pánico $(2 \%)$, sugiriendo que la SA explica, como mucho, solo una pequeña parte del desarrollo de AP inesperados.

El reciente estudio de Drost et al. (2012) estudia tres factores cognitivos, entre los que se encuentra la SA, para determinar la validez incremental de estos factores para predecir los trastornos de ansiedad (incluyendo TP) y el estado de ánimo. Sus resultados indican que, eliminando la influencia de los rasgos de personalidad Neuroticismo y Extraversión, la SA es un componente específico relacionado con el TP, específicamente la subescala física del ASI.

\section{La percepción de control de la ansiedad}

Barlow (2002) describe la ansiedad como un proceso cognitivo-afectivo donde el individuo tiene un sentido de impredictibilidad e incontrolabilidad de los acontecimientos y emociones potencialmente negativas y perjudiciales. Este sentido se asocia con activación fisiológica, aprensión ansiosa e incertidumbre sobre la habilidad de manejar los miedos. Parece existir suficiente evidencia empírica sobre la influencia de la percepción de control en los modelos del TP (Barlow, 2002; White y Barlow, 2002). Desde esta perspectiva, los individuos con TP a menudo piensan que no pueden contro- 
lar eficazmente sus experiencias emocionales o las situaciones vitales complicadas (Rapee, Craske, Brown y Barlow, 1996) y utilizan drásticos esfuerzos para prevenir o reducir temporalmente sus estados de ansiedad (Cox, Endler, Swinson y Norton, 1992; Zvolensky, Bernstein, Marshall y Feldner, 2006). Esto iría en la línea de los hallazgos que indican que los pacientes con TP tienen déficits en el procesamiento emocional, lo cual se considera un factor de vulnerabilidad para el desarrollo del primer AP (Baker et al., 2004).

Un aspecto específico relacionado con la percepción de control es la percepción de control de la ansiedad (PCA). Dada la relevancia que la regulación emocional tiene en la etiología y mantenimiento del TP, la investigación reciente se focaliza en el estudio de la influencia, el efecto modulador y el valor predictivo de la PCA en variables clínicas relevantes en el TP como la evitación agorafóbica (White, Brown, Somers y Barlow, 2006), el miedo a las sensaciones físicas (Gregor y Zvolensky, 2008) o el ajuste psicológico (Sokolowski e Israel, 2008). La PCA es una medida a través de la cual los miedos externos y las reacciones emocionales individuales se consideran bajo nuestro propio control. White et al. (2006), en una muestra de 239 pacientes con TP encontraron que niveles bajos de PCA medidos con el Anxiety Control Questionnaire- Revised (ACQ-R; Brown, White, Forsyth y Barlow, 2004) se asociaron con una fuerte relación entre SA y AG. Se puede decir que un individuo con alta SA y puntuaciones elevadas en PCA hacia síntomas físicos indeseables puede tener menor riesgo de respuesta con sintomatología de pánico. En ese caso, la PCA se convierte en un recurso psicológico adecuado para tolerar o afrontar los estresores (Gregor y Zvolensky, 2008).

A pesar de que la SA y la PCA se han propuesto como los dos constructos explicativos más importantes en los modelos de vulnerabilidad en pánico, no actúan de la misma manera. Cuando se consideran ambos en el mismo modelo general, la SA es el mejor predictor comparado con la PCA. De acuerdo con Zvolensky et al. (2006), desde una perspectiva preventiva, sería más práctico y productivo identificar altos niveles en SA que altos niveles en PCA para la identificación de personas en riesgo. Aún así, Brown et al. (2004) ponen de manifiesto que, en experimentos de laboratorio en los que se inducía pánico, las personas con TP mostraban significativamente menos miedo cuando percibían que el procedimiento estaba controlado (Rapee, Mattick y Murrell, 1986; Sanderson, Rapee y Barlow, 1989). El estudio de Zebb y Moore (2003) indicó que la PAC se asocia con trastornos de ansiedad específicos (p. e. TP, Trastorno de Ansiedad Generalizada, Trastorno Obsesivo-Compulsivo y Fobia Social), y con medidas generales de estrés psicológico (ansiedad y depresión). La PAC se asocia negativamente con la tendencia a responder con miedo a los síntomas de ansiedad, lo cual está relacionado teóricamente con el aumento de esos síntomas y la consecuencia de la espiral hacia la ansiedad o el pánico (Forsyth, Parker y Finlay, 2003).

\section{Conclusiones}

A lo largo de este artículo hemos descrito los resultados obtenidos por distintos investigadores con respecto a la relación entre personalidad y el TP, con o sin AG.

Los resultados obtenidos por nuestra revisión (Tabla 1) indican, al igual que otros autores, que la prevalencia de los TPe en pacientes con TP/TPA es elevada (Albert et al., 2006; Iketani et al., 2002; Iketani et al., 2004; Latas et al., 2000; Marchesi et al., 2005; Marchesi et al., 2006; Ozkan y Altindag, 2005; Venturello et al., 2002). Los resultados obtenidos respecto a la identificación de Clusters y TPe específicos coindiden en parte con el estudio de metanálisis recientemente publicado de Friborg, Martinussen, Kaiser, Overgard y Rosenvinge (2013). Estos autores concluyen que, el Cluster $\mathrm{C}$ de los TPe es el que mayor proporción obtiene en muestras de trastornos de ansiedad en general y específicamente en TP/TPA, seguido del B y el A. Respecto a los TPe específicos que obtienen una mayor proporción según este trabajo son el TPe Evitativo (TP: .15 y TPA: .17) y el TPe Dependiente (ambos .13). Estos resultados coinciden con los obtenidos en la presente revisión cuando tenemos en cuenta la muestra de pacientes con TPA. Al considerar la muestra de TP o la conjunta, el TPe Obsesivo-compulsivo emerge como el más prevalente seguido del TPe Evitativo en la muestra conjunta y el TPe Paranoide en TP. Algunos estudios relacionan la variable del perfeccionismo y el control con el TP (Antony et al., 1998; Iketani et al., 2002), variables características en el TPe Obsesivo-compulsivo.

Estos resultados subrayan las diferencias entre los perfiles de personalidad de los pacientes con TP y aquellos con TPA. Según nuestra revisión, desde el enfoque de personalidad clínico, los pacientes con TP obtienen mayores porcentajes de casos en el TPe Obsesivo-compulsivo del Cluster C y en el TPe Paranoide del Cluster A, sin embargo, los pacientes con TPA obtienen porcentajes mayores en el TPe Evitativo y Dependiente del Cluster C, en todos los TPe del Cluster B y en los TPe Esquizoide y Esquizotípico. Estos resultados coinciden, con los obtenidos por otros autores (Alnaes y Torgersen, 1988a; Alnaes y Torgersen, 1988b; Alnaes y Torgersen, 1990; Iketani et al., 2002; Mavissakalian y Hamman, 1988).

Parece evidenciarse que los pacientes con TPA poseen una mayor tendencia a la evitación y la dependencia. Estos resultados apoyarían las caracterizaciones clásicas de los pacientes con TPA que los describían como excesivamente ansiosos, evitadores, dependientes, no asertivos y con falta de confianza (Chambles, 1982; Mathews, Gelder y Johnston, 1981; Thorpe y Burns, 1983). Por el contrario, los pacientes con TP parecen caracterizarse además de por la evitación, por el pensamiento rígido y perfeccionista propio del TPe Obsesivo-compulsivo, así como por un pensamiento más suspicaz y desconfiado hacia los demás. Este perfil de personalidad del TP explicaría los datos que indican que los pacientes con TP y TPe manifiestan mayor sintomatología y peores puntuaciones en funcionamiento social (Chambless 
et al., 1992; Ozkan y Altindag, 2005; Reich et al., 1994; Segui et al., 1999; Seivewright et al., 2004).

Desde enfoques de personalidad normal también se encuentran diferencias entre muestras de TP o TPA, aunque ambos puntúan alto en Neuroticismo y bajo en emociones positivas, los pacientes con AG puntúan bajo en el factor Extraversión, sobre todo en la faceta de cordialidad y en la faceta confianza de la dimensión Amabilidad. Los pacientes con TP también puntúan bajo en autodisciplina, faceta del factor Responsabilidad (Bienvenu et al., 2004).

Se han descrito variables que influyen en el aumento de la prevalencia de los TPe en el TP como por ejemplo la evitación fóbica (Friedman et al., 1987), la presencia de AG (Iketani et al., 2002), los síntomas de depresión (Alnaes y Torgersen, 1990; Chambless et al., 1992; Klass et al., 1989; Reich y Troughton, 1988; Reich et al., 1994; Ozkan y Altindag, 2005) o la edad de inicio del trastorno (menos de 18 años). Los pacientes clasificados en este subgrupo obtienen mayor comorbilidad con TPe, concretamente el Cluster C, tienen al menos un diagnóstico más en Eje I y mayores niveles de ansiedad y depresión (Venturello et al., 2002), mayor gravedad en los síntomas y aumento de la prevalencia de la AG (Battaglia et al., 1997; Iketani et al., 2004; Ozkan y Altindag, 2005). En conclusión, parece que la patología de personalidad se encuentra entre las variables que aumentan la gravedad del TP/TPA.

Respecto a las diferencias en función del sexo, parece que distintos autores han encontrado mayores porcentajes de TPe Paranoide, TPe Esquizoide (Reich et al., 1994; Barzega et al., 2001), TPe Evitativo y TPe Compulsivo (Reich y Troughton, 1987) en hombres y TPe Histriónico en mujeres (Barzega et al., 2001). Es necesaria mayor investigación respecto a esta variable en muestras con TP/TPA.

Existe suficiente evidencia científica para decir que los factores o dimensiones de personalidad más relacionadas con el TP/TPA son el Neuroticismo elevado y puntuaciones bajas en Extraversión y Responsabilidad (Bienvenu et al., 2001, 2004; Carrera et al., 2006; Kotov et al., 2010). Consideramos importante mencionar que al considerar las facetas de personalidad de segundo orden podemos encontrar perfiles con mayor especificidad en el conjunto de trastornos de ansiedad y del estado de ánimo. En este sentido, los pacientes con TP y TPA coinciden en obtener puntuaciones bajas en las facetas confianza, actitud conciliadora, autodisciplina y cordialidad, sin embargo, los pacientes con TP obtienen a su vez puntuaciones bajas en las facetas emociones positivas, asertividad y competencia (Bienvenu et al., 2001, 2004). Nuevos estudios serán necesarios para acumular evidencia respecto al perfil diferencial en pacientes con TP y TPA a través del estudio de las facetas de personalidad de segundo orden.

Respecto a los factores de riesgo o vulnerabilidad identificados por la comunidad científica en muestras con TP/TPA se concluye que, en relación a las variables parentales y diagnósticos de ansiedad en la infancia, los padres y madres sobreprotectores y rígidos (Latas et al., 2000), expe- rimentar eventos estresantes como problemas económicos, abuso o pérdida temprana de padres durante la infancia y adolescencia (Kendler et al., 2000a; Moisan y Engels, 1995; Reti et al., 2002 a,b) o sufrir ansiedad por separación en la infancia (Venturello et al., 2002) se relacionan con la mayor probabilidad de desarrollar y mantener tanto TP o TPA como TPe en la edad adulta. Los estudios longitudinales sugieren que la IC estaría relacionada con el desarrollo de trastornos emocionales pero falta especificidad para considerarlo factor de riesgo para el TP (Biederman et al., 1993; Caspi et al., 1996; Eaton et al., 1994; Schwartz et al., 1999). Los datos parecen apoyar la base genética relacionada con el desarrollo de psicopatología internalizante (Brown y Barlow, 2009). Con respecto a la $\mathrm{SA}$, un número importante de estudios desde distintas aproximaciones han mostrado evidencia empírica de su relación con el TP (Brown et al., 2003; Penava et al., 1998; Plehn y Peterson, 2002; Simon et al., 2004; Telch et al., 2003). Respecto a la PCA, a pesar de ser una línea de investigación más reciente, ya parece contar con evidencias empíricas sobre su influencia, su efecto modulador o su valor predictivo respecto a variables clínicas en el TP como la evitación agorafóbica, el miedo a las sensaciones físicas y el ajuste psicológico (Gregor y Zvolensky, 2008; Sokolowski e Israel, 2008; White, et al., 2006).

\section{Aplicabilidad social y clínica de los resultados obte- nidos}

La identificación de factores de riesgo podría servir, en primer lugar, para conocer los factores que influyen en la mayor probabilidad de desarrollar patología clínica en la infancia, adolescencia o edad adulta y, por tanto, podrían orientarnos en el desarrollo de programas de promoción de la salud mental en población infanto-juvenil (Puura et al. 2002). En vista de los resultados, parece clara la necesidad de que en los niños/as se fomente y se entrene en estrategias de regulación emocional. Las intervenciones deben incluir a todos los agentes implicados por lo que, en la edad infantojuvenil, sería imprescindible trabajar en el contexto familiar y escolar (Olweus, 2004). Cabe mencionar que intervenciones de estas características ya han dado resultados de eficacia en los contextos escolares (Domitrovich, et al., 2010; Hosman, Dadds y Raphael, 2005; Greenberg, Domitrovich y Bumbarger, 2001).

Por otra parte, conseguiríamos la identificación de factores de vulnerabilidad en edad temprana pudiendo ayudar a padres e incluso a maestros, en la detección precoz y en cómo actuar ante estos niños/ as vulnerables. En este caso es donde tendrían cabida las intervenciones preventivas secundarias, cuyo objetivo sería la intervención en grupos específicos vulnerables con el objetivo de no desarrollar TP/TPA o rasgos desadaptativos de personalidad.

Finalmente, en el caso de presentarse psicopatología ansiosa o depresiva en la infancia, conocer los factores de riesgo, podría ayudarnos a mejorar los programas de intervención incluyendo elementos terapéuticos específicos en fun- 
ción de la sintomatología clínica, de la personalidad y de las variables contextuales (estilos educativos parentales, etc.). Los conocimientos sobre la influencia de la personalidad en el inicio, curso y pronóstico del TP/TPA podrían mejorar también los programas de tratamiento cognitivocomportamentales para adultos existentes, aumentando la adherencia y la satisfacción de los pacientes, reduciendo en menor tiempo y con mayor estabilidad la sintomatología ansiosa, la interferencia, el malestar que ocasiona y el consumo de fármacos. El ajuste de los tratamientos a las necesidades del paciente no estaría reñida con la protocolización de los programas de intervención. El objetivo es incorporar a los programas de tratamiento las herramientas necesarias en función de las características de los pacientes con TP/TPA.

Los resultados que hemos descrito en este trabajo parecen indicar que las dimensiones y rasgos de personalidad patológicos más relacionados con el constructo de emocionalidad negativa y desregulación emocional (neuroticismo, inhibición comportamental, rasgos evitativos, dependientes $\mathrm{u}$ obsesivos, etc.) se asocian con el TP, con o sin AG. Además, parece que ese constructo es compartido por otros trastornos de ansiedad y afectivos. En este sentido, nos gustaría mencionar la propuesta de una clasificación dimensional de los trastornos emocionales realizada por Brown y Barlow (2009). Estos autores consideran dos dimensiones de temperamento independientes y genéticamente determinadas como base de todos los trastornos emocionales: el neuroticismo o emocionalidad negativa y la extraversión o emocionalidad positiva. Estas dimensiones estarían en el nivel más alto y se relacionarían con los constructos de Gray (1987): Sistema de Inhibición Comportamental y Sistema de Activación Comportamental. El individuo con alto neuroticismo tendría una tendencia a manifestar conductas de evitación, incluyendo evitación conductual e interoceptiva y evitación cognitiva y emocional. Esta vulnerabilidad, asociada a acontecimientos vitales estresantes llevaría a distintas manifestaciones psicopatológicas entre las que incluyen AP, síntomas somáticos, cogniciones intrusivas, miedo a la evaluación negativa o síntomas postraumáticos. En resumen, esta propuesta considera que todos los trastornos emocionales comparten temperamentos comunes como factores de vulnerabilidad, que, asociados a experiencias vitales de distinta índole llevarán a las diferentes manifestaciones que constituirían los distintos trastornos, entre los que se incluye el TP, con o sin AG. Para concluir, estos prestigiosos autores señalan la importancia de la personalidad en la comprensión del TP/TPA e indican la importancia de tenerla en cuenta en la conceptualización y abordaje de cualquiera de los trastornos emocionales.

Los resultados que hemos mencionado respecto a los factores de vulnerabilidad para el desarrollo de patología en Eje I (TP/TPA) y II (Cluster C), parecen guardar relación con los problemas de regulación emocional que se observan en la población clínica (Hervás, 2011). En concreto, estos problemas se han encontrado en pacientes con trastornos depresivos, en la mayoría de los trastornos de ansiedad y en los trastornos de la personalidad. Las personas que sufren estos trastornos presentarían déficits de regulación emocional, es decir, desregulación afectiva. La investigación en regulación emocional y psicopatología indica que la eficacia de la terapia cognitivo-comportamental se podría mejorar identificando las habilidades generales de regulación emocional, desarrollando intervenciones que puedan mejorar estas habilidades e incluyendo estas intervenciones en los programas terapéuticos. Estos han sido los objetivos que han guiado al diseño y elaboración del protocolo de tratamiento unificado para los trastornos emocionales (Barlow et al., 2011).

Basado en los resultados de la investigación, Barlow et al. (2011) han desarrollado un tratamiento aplicable a todos los trastornos de ansiedad y los del estado de ánimo unipolar, y potencialmente valido para otros trastornos con un fuerte componente emocional o somatomorfo. Este protocolo unificado incorpora las técnicas psicológicas que hasta el momento han demostrado empíricamente su eficacia, como por ejemplo: la reevaluación de las interpretaciones cognitivas desadaptativas, cambios en las tendencias de acción asociadas con los trastornos emocionales, prevención de la evitación emocional, y la utilización de procedimientos de exposición emocional. Este protocolo ya ha sido sometido a prueba consiguiendo beneficios terapéuticos como mínimo en la misma medida que los conseguidos por los tratamientos cognitivo-comportamentales (Ellard et al., 2010).

\section{Líneas futuras}

Para concluir, consideramos que las líneas futuras de investigación interesadas en el ámbito de la personalidad y su relación con el TP/TPA, seguirán estudiando factores de segundo orden que puedan delimitar perfiles clínicos específicos entre los trastornos. También observaremos un creciente interés por los aspectos psicopatológicos y psicosociales que subyacen y son comunes a los trastornos emocionales y a los rasgos y TPe. Los enfoques dimensionales ofrecerán nuevas alternativas de evaluación, diagnóstico y conceptualización de los trastornos emocionales y de la personalidad (algunos cambios podrán observarse ya en el DSM-5). Finalmente, los aspectos emocionales cobrarán mayor relevancia tanto a nivel teórico como de intervención, convirtiéndose en aspectos centrales en los programas de tratamiento cognitivocomportamentales indicados para el tratamiento de trastornos emocionales y de la personalidad.

Agradecimientos.- Financiado por Gobierno de Aragón (Dpt. Industria e Innovación), Fondo Social Europeo y Ministerio de Economía y Competitividad, Plan Nacional (PSI201021423/PSIC). 


\section{Referencias}

Albert, U., Maina, G., Bergesio, C. y Bogetto, F. (2006). Axis I and II comorbidities in subjects with and without nocturnal panic. Depression and Anxiety, 23, 422-428.

Alnaes, R. y Torgersen, S. (1988a). DSM-III symptom disorders (Axis I) and personality disorders (Axis II) in an outpatient population. Acta Psychiatrica Scandinavica, 78, 348-355.

Alnaes, R. y Torgersen, S. (1988b). The relationship between DSM-III symptom disorders (Axis I) and personality disorders 8Axis II) in an outpatients population. Acta Psychiatrica Sacandinavica, 78, 435-492.

Alnaes, R. y Torgersen, S. (1990). MCMI Personality Disorders Among Patients With Major Depresion With And Without Anxiety Disorders. Journal of Personality Disorders, 4, 141-149.

Ampollini, P., Marchesi, C., Signifredi, R., Ghinaglia, E., Scardovi, F., Codeluppi, S. et al. (1999). Temperament and personality features in patients with major depression, panic disorder and mixed conditions. Journal of Affective Disorders, 52, 203-207.

Antony, M. M., Purdon, C. L., Huta, V. y Swinson R. P. (1998). Dimensions of perfectionism across the anxiety disorders. Behaviour Research and Therapy, 36, 1143-54.

Aronson, T. A. y Logue, C. M. (1987). On the longitudinal course of panic disorder: development history and predictors of phobic complications. Comprehensive Psychiatry, 28, 344-355.

Asmundson, G. J. y Norton, G. R. (1993). Anxiety sensitivity and its relationship to spontaneous and cued panic attacks in collage students. Behaviour Research and Therapy, 31, 199-201.

Baker R., Holloway J., Thomas P.W., Thomas S., Owens M. (2004). Emotional processing and panic. Behaviour Research and Therapy, 42, 11, 12711287

Barlow, D. H. (2002). Anxiety and its disorders: The nature and treatment of anxiety and panic ( $\left.2^{\mathrm{a}} \mathrm{ed}.\right)$. New York, NY: Guilford Press.

Barlow, D. H., Farchione, T.J., Fairholme, C.P., Ellard, K.K., Boisseau, C. L. Allen, L. B. y Ehrenreich-May, J. (2011). The unified protocol for transdiagnostic treatment of emotional disorders: Therapist guide. New York, NY: Oxford University Press.

Barzega, G., Maina, G., Venturello, S. y Bogetto, F. (2001). Gender-related distribution of personality disorders in a sample of patients with panic disorder. European Psychiatry, 16, 173-179.

Battaglia, M., Bajo, S., Strambi, L. F., Brambilla, F., Castronovo, C., Vanni, G. et al. (1997). Physiological and behavioral responses to minor stressors in Offspring of patients with panic disorder. Journal of Psychiatric Research, 31, 365-376.

Berger, P., Sachs, G., Amering, M., Holzinger, A., Bankier, B. y Katschnig, H. (2004). Personality disorder and social anxiety predict delayed response in drug and behavioral treatment of panic disorder. Journal of $A f$ fective Disorders, 80, 75-78.

Biederman, J., Rosenbaum, J. F., Bolduc-Murphy, E. A., Faraone, S. V., Chaloff, J., Hirshfeld, D. R., et al. (1993). A 3-year follow-up of children with and without behavioral inhibition. Journal of the American Academy of Child and Adolescent Psychiatry, 32, 814-821.

Bienvenu, O. J., Nestadt, G., Samuels, J. F., Costa, P. T., Howard, W. T. y Eaton, W. W. (2001). Phobic, panic, and major depression disorders and the five-factor modelo f personality. The Journal of Nervous and Mental Disease, 189, 154-161.

Bienvenu, O. J., Samuels, J. F., Costa, P. T., Reti, I. M., Eaton, .W. W. y Nestadt, G. (2004). Anxiety and depressive disorders and the five-factor model of personality: a higher-and lower- order personality trait investigation in a community sample. Depression and Anxiety, 20, 92-97.

Bienvenu, O. J., Hettema, J. M., Neale, M. C., Prescott, C. A. y kendler, K. S. (2007). Low extraversión and high neuroticismo as indices of genetic and environmental risk for social phobia, agoraphobia, and animal phobia. The American Journal of Psychiatry, 164, 1714-1721.

Bramsen, I., Dirkzwager, A. J. y van der Ploeg, H. M. (2000). Predeployment personality traits and exposure to trauma as predictors of posttraumatic stress symptoms: a prospective study of former peacekeepers. American Journal of Psychiatry, 157, 1115-1119.

Brooks, R. B., Baltazar, P. L., McDowell, D. E., Munjack, D. J. y Bruns, J. R. (1988). Personality disorders co-ocurring with panic disorder with agoraphobia. Journal of Personality Disorders, 5, 326-328.
Brown, T. A y Barlow, D. H. (1992). Comorbidity among anxiety disorders: Implications for treatment and DSM-IV. Journal of Consulting and Clinical Psychology, 60, 835-844.

Brown, T. A. y Barlow, D. H. (2009). A proposal for a dimensional classification system based on the shared features of the DSM-IV anxiety and mood disorders: Implications for assessment and treatment. Psychological Assessment, 21(3), 256-271.

Brown, G. W., Harris, T. O. y Eales, M. J. (1996). Social factors and comorbidity of depressive and anxiety disorders. British Journal of Psychiatry, $168,50-57$.

Brown, M., Smits, J. A., Powers, M. B. y Telch, M. J. (2003). Differential sensitivity of the three ASI factors in predicting panic disorder patients'subjective and behavioural response to hyperventilation challenge. Journal of Anxiety Disorders, 17, 583-591.

Brown, T. A., White, K. S., Forsyth, J. P. y Barlow, D. H. (2004). The structure of perceived emotional control: Psychometric properties of a revised Anxiety Control Questionnaire. Behavior Therapy, 35, 75-99.

Carrera, M, Herrán, A., Ramírez, M. L., Ayestarán, A., Sierra-Biddle, D., Hoyuela, F., Rodríguez-Cabo, B. y Vazquez-Barquero, J. L. (2006). Personality traits in early phases of panic disorder: implications on the presence of agoraphobia, clinical severity and short-term outcome. Acta Psychiatrica Scandinavica, 114, 417-425.

Caspi, A. y Silva, P. A. (1995). Temperamental qualities at age three predict personality traits in young adulthood: Longitudinal evidence from a birth cohort. Child Development, 66, 486-498.

Caspi, A., Moffitt, T. E., Newman, D. L. y Silva, P. A. (1996). Behavioral observations at age 3 years predict adult psychiatric disorders: Longitudinal evidence from a birth cohort. Archives of General Psychiatry, 53, 1033-1039.

Chambless, D. L. (1982). Characteristics of agoraphobics. In Chambless D. L., Goldstein, A. J. (Eds.), Agoraphobia: Multiple Perspectives on Theory and Treatment. New York, John Wiley \& Sons.

Chambless, D. L. y Renneberg, B. (1988). Personality disorders of agoraphobics. Paper presented at World Congress of Behavior Therapy, Edinburgh, Scotland.

Chambles, D. L., Renneberg, B. y Goldstein, A. (1992). MCMI-diagnosed personality disorders among agoraphobic outpatients: Prevalence and relationship to severity and treatment Outcome. Journal of Anxiety Disorders, 6, 193-211.

Clark, L. A. y Watson, D. (1991). Tripartite model of anxiety and depression: Psychometric evidence and taxonomic implications. Journal of Abnormal Psychology, 100, 316-336.

Costa, P. y McCrae, R. (1980). Influences of extraversion and neuroticism on subjective well-being: Happy and unhappy people. Journal of Personality and Social Psychology, 38, 668-678.

Cox, B., Endler, N., Swinson, R. y Norton, G. (1992). Situations and specific coping strategies associated with clinical and nonclinical panic attacks. Behaviour Research and Therapy, 30, 67-69.

Cox, B. J., Enns, M. W., Walker, J. R., Kjernisted, K. y Pidlubny, S. R. (2001). Psychological vulnerabilities in patients with major depression vs panic disorder. Behaviour Research and Therapy, 3, 567-573.

Domitrovich, C. E., Bradshaw, C. P., Greenberg, M. T., Embry, D., Poduska, J. M. y Ialongo, N. S. (2010). Integrated models of school-based prevention: Logic and Theory. Psychology in the Schools, 47 (1), 71-88.

Drost, J., Van der Does, A. J. W., Antypa, N., Zitman, F. G., Van Dyck, R. y Spinhoven, Ph. (2012). General, specific and unique cognitive factors envolved in anxiety and depresión disorders. Cognitive Therapy and Research, 36, 621-633.

Eaton, W. W., Kessler, R. C., Wittchen, H. U. y Magee, W. J. (1994). Panic and panic disorder in the United States. American Journal of Psychiatry, 41, 413-420.

Eysenck, M. (1982). Attention and arousal. New York: Springer.

Ellard, K. K., Fairholme, C. P., Boisseau, C. L., Farchione, T. J. y Barlow, D. H. (2010). Unified Protocol for the transdiagnostic treatment of emotional disorders: Protocol development and initial outcome data. Cognitive and Behavioral Practice, 17, 88-101.

Faravelli, C. y Albanesi, G. (1987). Agoraphobia with panic attacks: 1-year prospective follow-up. Comprehensive Psychiatry, 28, 481-7. 
Fauerbach, J. A., Lawrence, J. W., Schmidt, C. W., Munster, A. M. y Costa, P. T. (2000). Personality predictors of injury-related posttaumatic stress disorder. Journal of Nervous and Mental Disease, 188, 510-517.

Foot, M. y Koszycki, D. (2004). Gender differences in anxiety-related traits in patients with panic disorder. Depression and Anxiety, 20,123-130.

Forsyth, J. P., Parker, J. D. y Finlay, C. G. (2003). Anxiety sensitivity, controllability, and experiential avoidance and their relation to drug of choice and addiction severity in a residential sample of substanceabusing veterans. Addictive Behaviors, 28, 851-870.

Friborg, O., Martinussen, M., Kaiser, S., Overgard, K. T. y Rosenvinge, J. H. (2013). Comorbidity of personality disorders in anxiety disorders: A meta-analysis of 30 years of research. Journal of Affective Disorders, 145, 143155.

Friedman, C. J., Shear, M. K. y Frances, A. (1987). DSM-III personality disorders in panic patients. Journal of personality Disorders, 1, 132-135.

Frost, R. O. y Steketee, G. (1997). Perfectionism in obsessive-compulsive disorder patients. Behaviour Research and Therapy, 35, 291-296.

García-Coll, C., Kagan, J. y Reznick, J. S. (1984). Behavioral inhibition in young children. Child Development, 55, 1005-1019.

Gerlsma, C., Emmelkamp, P. M. G. y Arrindell, W. A. (1990). Anxiety, depression, and perception of early parenting: a meta-analysis. Clinical Psychology Review, 10, 251-257.

Gittelman, R. y Klein, D. F. (1984). Relationship between separation anxiety and panic and agoraphobic disorders. Psychopathology, 17, 56-65.

Gorman, J. M., Papp, L. A., Coplan, J. D., Martínez, J. M., Lennon, S., Goetz, R. R. et al. (1994). Anxiogenic effects of CO2 and hyperventilation in patients with panic disorder. American Journal of Psychiatry, 151, 547-553.

Grant, B. F., Hasin, D. S., Stinson, F. S., Dawson, D. A., Patricia Chou, S. June Ruan W. y Huang, B. (2005). Co-occurrence of 12-month mood and anxiety disorders and personality disorders in the US: results from the national epidemiologic survey on alcohol and related conditions. Journal of Psychiatric Research, 39, 1-9.

Gray, J. A. (1987). The psychology of fear and stress (2nd ed.). Cambridge, England: Cambridge University Press.

Green, M. A. y Curtis, G. C. (1988). Personality disorders in panic patients: Response to termination of antipanic medication. Journal of Personality Disorders, 2, 303-314.

Greenberg, M. T., Domitrovich, C. y Bumbarger, B. (2001). The prevention of mental disorders in school-aged children: Current state of the field. Prevention \& Treatment, 4(1), Mar 2001, No Pagination Specified Article 1 a.

Gregor, K. L. y Zvolensky, M. J. (2008). Anxiety sensitivity and perceived control over anxiety-related events: Evaluating the singular and interactive effects in the prediction of anxious and fearful responding to bodily sensations. Bahaviour Research and Therapy, 46, 1017-1025.

Hayward, G., Killen, J. D., Kraemer, H. C. y Taylor, C. B. (2000). Predictors of panic attacks in adolescents. Journal of the American Academy of Child and Adolescent Psychiatry, 39, 207-214.

Hervás, G. (2011). Psicopatología de la regulación emocional: el papel de los déficits emocionales en los trastornos clínicos. Psicología Conductual, 19, 347-372.

Hoffart, A. y Hedley, L. M. (1997). Personality traits among panic disorder with agoraphobia patients before and after symptom-focused treatment. Journal of Anxiety Disorders, 11, 77-87.

Hosman, C., Dadds, M. y Raphael, B. (2005). Prevención de los trastornos de ansiedad. En: Hosman, C., Jané-Llopis, E. y Saxena, S. (Eds.), Prevención de trastornos mentales: intervenciones efectivas y opciones de politicas. Oxford, Oxford University Press.

Iketani, T., Kiriike, N., Stein, M. B., Nagao, K., Nagata, T., Minamikawa, N., Shidao, A. y Fukuhara, H. (2002). Relationship between perfectionism, personality disorders and agoraphobia in patients with panic disorder. Acta Psychiatrica Scandinavica, 106, 171-178.

Iketani, T., Kiriike, N., Stein, M. B., Nagao, K., Minamikawa, N, Shidao, A. y Fukuhara, H. (2004). Patterns of Axis II Comorbidity in Early-Onset Versus Late-Onset Panic Disorder in Japan. Comprehensive Psychiatry, 45, 114-120

Jankowsky, D. S., Morter, S. y Tancer, M. (2000). Over-representation of Myers Briggs Type Indicator introversion in social phobia patients. Depression and Anxiety, 11, 121-125.
Johnson, J. G., Cohen, P., Brown, J., Smailes, E. M. y Bernstein, D. P. (1999). Childhood maltrearment increases risk for personality disorders during early adulthood. Archives of General Psychiatry, 56, 600-606.

Kagan, J., Snidman, N., Arcus, D. y Reznick, J. S. (1994). Galen's prophecy: Temperament in human nature. New York: Basic Books.

Karsten, J., Penninx, B. W. J. H., Riese, H., Ormel, J., Nolen, W. A. y Hartman, C. A. (2012). The state effect of depressive and anxiety disorders on big five personality traits. Journal of Psychiatric Research, 46, 644-650.

Kasen, S., Cohen, P., Skodol, A. E., Johnson, J. G., Smailes, E. y Brook, J. S. (2001). Childhood depression and adult personality disorder: alternative pathways of continuity. Archives of General Psychiatry, 58, 231-236.

Kendler, K. S., Bulik, C. M., Silberg, J., Hettema, J. M., Myers, J. y Prescott, C. A. (2000a). Childhood sexual abuse and adult psychiatric and substance use disorders in women: An epidemiological and cotwin control analysis. Archives of General Psychiatry, 57, 953-959.

Kendler, K. S., Myers, J. y Prescott, C. A. (2000b). Paenting and adult mood, anxiety, and substance use disorders in female twins: An epidemiological, multi-informant, retrospective study. Psychological Medicine, 30, 281294.

Klass, E. T., DiNardo, P. A. y Barlow, D. H. (1989). DSM-III-R personality diagnosis in anxiety disorder patients. Comprehensive Psychiatry, 30, $251-$ 258.

Klein, D. F. (1964). Delineation of two drug responsive anxiety syndromes. Psychofarmacologia, 5, 397-408.

Koenigsberg, H. W., Kaplan, R. D., Gilmore, M. M. y Cooper, A. M. (1985). The relationship between syndrome and personality disorder in DSMIII: Experience with 2,462 patients. American Journal of Psychiatry, 142, 207.

Kotov, R., Gamez, W., Schmidt F. y Watson, D. (2010). Linking "big" personality traits to anxiety, depressive, and substance use disorders: A meta-analysis. Psychological Bulletin, 136 (5), 768-821.

Langs, G., Quehenberger, F., Fabisch, K., Klug, G., Fabisch, H. y Zapotoczky, H. G. (1998). Prevalence, pattern and role of personality disorders in panic disorder patients with and without comorbid (lifetime) major depression. Acta Psychiatrica Sacandinavica, 98, 116-123.

Latas, M. Starcevic, V., Trajkovic, G. y Bogojevic, G. (2000). Predictors of Comorbid Personality disorders in patients with panic disorder with agoraphobia. Comprehensive Psychiatry, 41, 28-34.

Lepine, J. P., Chignon, J. M. y Teherani, M. (1993). Suicide attempts in patients with panic disorder. Archives of General Psychiatry, 50, 144-149.

Lewinsohn, P., Zinbarg, R., Seeley, J., Lewinsohn, M. y Sack, W. (1997). Comorbidity between anxiety disorders and between anxiety disorders and other mental disorders in children. Journal of Anxiety Disorders, 11 , 377-394.

Maller, R. G. y Reiss, S. (1992). Anxiety sensitivity in 1984 and panic attacks in 1987. Journal of Anxiety Disorders, 6, 241-247.

Manassis, K., Bradley, S., Goldberg, S., Hood, J. y Swinson, R. P. (1995). Behavioural inhibition, attachment and anxiety in children of mothers with anxiety disorders. Canadian Journal of Psychiatry. Revue Canadienne de Psychiatrie, 40, 87-92.

Marchand, A., Goyer, L. R., Dupuis, G. Y Mainguy, N. (1998). Personality disorders and the outcome of cognitive-behavioural treatment of panic disorder with agoraphobia. Canadian Journal of Behavioural Science, 30, 14 23

Marchesi, C., Cantón, A., Fonito, S., Giannelli, M. R. y Maggini, C. (2005). The effect of pharmacotherapy on personality disorders in panic disorder: A one year naturalistic study. Journal of Affective Disorders, 89, 189 194

Marchesi, C., De Panfilis, C., Cantón, A., Fonito, S., Giannelli, M. R. y Maggini, C. (2006). Personality disorders and response to medication treatment in panic disorder: A 1-year naturalistic study. Progress in NeuroPsychopharmacology \& Biological Psychiatry, 30, 1240-1245.

Massion, A. O., Dyck, I. R., Shea, M. T., Phillips, K. A., Warshaw, M. G. y Keller, M. B. (2002). Personality disorders and time to remission in generalized anxiety disorder, social phobia, and panic disorder. Archives of General Psychiatry, 59, 434-440.

Mathews, A. M., Gelder, M.G. y Johnston, D. W. (1981). Agoraphobia: Nature and treatment. New York: Guilford Press. 
Matsunaga, H., Iketani, T., Matsui, T., Oya, K., Iwasaki, Y. y Miyata, A. (2000). Perfectionism in patients with obsessive-compulsive disorder. Rinsho Seishin Igaku (in Japanese), 29, 1625-1632.

Mauri, M., Sarno, N. Rossi, V. M., Armani, A., Zambotto, S., Cassano, G. B. y Akiskal, H. S. (1992). Personality disorders associated with generalized anxiety, panic and recurrent depressive disorders. Journal of Personality Disorders, 6, 162-167.

Mavissakalian, M. R. y Hamann, M. S. (1986). Assessment and significance of behavioral avoidance in agoraphobia. Journal of Psychopathology and Behavioral Assessment, 8, 199-210.

Mavissakalian, M. R. y Hamann, M. S. (1987). DSM-III personality disorder in agoraphobia: II. Changes with treatment. Comprehensive Psychiatry, 28, 356-361.

Mavissakalian, M. R. y Hamann, M. S. (1988). Correlates of personality disorder in panic disorder and agoraphobia. Comprehensive Psychiatry, 29, 535-544.

Mendoza, L., Navinés, R., Crippa, J. A., Fagundo, A. B., Gutierrez, F., Nardi, A. E., Bulbena, A., Valdés, M. Y Martín-Santos, R. (2011). Depersonalization and personality in panic disorder. Comprehensive Psychiatry, 52, 413-419.

McNally, R. J. (2002). Anxiety sensitivity and panic disorder. Biological Psychiatry, 52, 938-946.

Milrod, B. L., Leon, A. C., Barber, J. P., Markowitz, J. C. y Graf, E. (2007). Do comorbid personality disorders moderate panic-focused psychotherapy? An exploratory examination of the American Psychiatric Association practice guideline. Jounal of Clinical Psychiatry, 68(6), 885-891.

Moisan, D. y Engels, M. L. (1995). Childhood trauma and personality disorder in 43 women with panic disorder. Psychological Report, 76, 1133-1134.

Nystrom, S. y Lindegard, B. (1975). Predisposition for mental syndromes: A study comparing predisposition for depression, neurasthenia and anxiety state. Acta Psicbiatrica Scandinavica, 51, 69-76.

Olweus, D. (2004). The Olweus Bullying Prevention Programe: Design and implementation issues and a new national initiative in Norway. In P. K. Smith, D. Pepler y K. Rigby (Eds.), Bullying in schools: How successful can interventions be? (pp. 13-36). Cambridge, UK: Cambridge University Press.

Ormel, J. y Wohlfarth, T. (1991). How neuroticism, long-term difficulties, and life situation change influence psychological distress: A longitudinal model. Journal of Personality and Social Psychology, 60, 744-755.

Ozkan, M. y Altindag, A. (2005). Comorbid personality disorders in subjects with panic disorder: do personality disorders increase clinical severity?. Comprehensive Psychiatry, 46, 20-26.

Osma, J. (2009). Influencia de la personalidad en la clínica del trastorno de pánico, con o sin AG: Un estudio exploratorio. (Tesis Doctoral, Universitat Jaume I). Recuperado de http://repositori.uij.es/xmlui/bitstream/handle/10234/9593/Influenci a personalidad.pdf? sequence $=1$.

Papp, L. A., Martínez, J. M., Klein, D. F., Coplan, J. D., Norman, R. G., Cole, R. et al. (1997). Respiratory psychophysiology of panic disorder: Three respiratory challenges in 98 subjects. American Journal of Psychiatry, $154,1557-1565$

Parker, G. y Gladstone, G. (1996). Parental characteristics as influences on adjustment in adulthood. In Pierce G. R., Saranson, B. R., Saranson, I. G. (Eds.), Handbook of Social Support and the Family. New York: NY: Plenum.

Penava, S. J., Otto, M. W., Maki, K. M. y Pollack, M. H. (1998). Rateo f improvement Turing cognitive-behavioral Group treatment for panic disorder. Behaviour Research and Therapy, 36, 665-673.

Plehn, K. y Peterson, R. A. (2002). Anxiety sensitivity as a predictor of the development of panic symptoms, panic attacks, and panic disorder: a prospective study. Journal of Anxiety Disorders, 16, 455-474.

Pollack, M. H., Otto, M. W., Rosenbaum, J. F. y Sachs, G. S. (1992). Personality disorders in patients with panic disorder. Comprehensive Psychiatry, 33, 78-83.

Poulton, R. y Andrews, G. (1992). Personality as a cause of adverse life events. Acta Psychiatrica Scandinavica, 85, 35-38.

Puura, K., Davis, H., Papadopoulou, K., Tsiantis, J., Ispanovic-Radojkovic, V., Rudic, N., Tamminem, T., Turunen, M., Dragonas, T., Paradisiotou, A., Vizakou, S., Roberts, R., Cox, A. y Day, C. (2002). The European early promotion project: a new primary health care service to promote children'smental health. Infant Mental Health, 22, 6, 606-624.
Rapee, R. M., Brown, T. A., Antony, M. M. y Barlow, D. H. (1992). Response to hyperventilation and inhalation of $5.5 \%$ carbon dioxideenriched air across the DSM-III-R anxiety disorders. Journal of Abnormal Psychology, 101, 538-552.

Rapee, R. M., Craske, M. G., Brown, T. A. y Barlow, D. H. (1996). Measurement of perceived control over anxiety-related events. Behavior Therapy, 27, 279-293.

Rapee, R., Mattick, R. y Murrell, E. (1986). Cognitive mediation in the effective component of spontaneous panic attacks. Journal of Behavior Therapy and Experimental Psychiatry, 17, 245-253.

Reich, J. (1987). Sex distribution of DSM-III personality disorders in psychiatric outpatients. American Journal of Psychiatry, 144, 485-488.

Reich, J. (1988). DSM-III personality disorders and the outcome of treated panic disorders. American Journal of Psychiatry, 145, 1149-1153.

Reich, J. (1991). Avoidant and dependent personality traits in relatives of patients with panic disorder, patients with dependent personality disorder, and normal controls. Psychiatry Research, 39, 89-98.

Reich, J. R. y Braginsky, Y. (1994). Paranoid Personality Traits in a Panic Disorder Population: A Pilot Study. Comprehensive Psychiatry, 35, 260-264.

Reich, J. R., Perry, J. C., Shera, D., Dyck, I., Vasile, R., Goisman, R. M., Rodriguez-Villa, F., Massion, A. O. y Keller, M. (1994). Comparison of personality disorders in different anxiety disorder diagnoses: Panic, Agoraphobia, Generalized anxiety, and Social Phobia. Annals of Clinical Psychiatry, 6, 125-134.

Reich, J. y Troughton, E. (1988). Frequency of DSM-III Personality Disorders in Patients With Panic Disorder: Comparison With Psychiatric and Normal Control Subjects. Psychiatry Research, 26, 89-100.

Reiss, S., Peterson, R. A., Gursky, D. M. y McNally, R. J. (1986). Anxiety sensitivity, anxiety frequency, and the prediction of fearfulness. Behaviour Research and Therapy, 24, 1-8.

Reti, I. M., Samuels, J. F., Eaton, W. W., Bienvenu, O. J., Costa, P. T. y Nestadt, G. (2002a). Influences of parenting normal personality traits. Psychiatry Research, 111, 55-64.

Reti, I. M., Samuels, J. F., Eaton, W. W., Costa, P. T., Bienvenu, O. J. y Nestadt, G. (2002b). Adult antisocial personality traits are associated with experiences of low parental care and maternal overprotection. Acta Psychiatrica Scandinavica, 106, 126-133.

Reznick, J. S., Hegeman, I. M., Kaufman, E. R. y Woods, S. W. (1992). Retrospective and concurrent self-report of behavioural inhibition and their relation to adult mental health. Development \& Psychopatology, 4, 301321.

Rosenbaum, J. F., Biederman, J., Bolduc-Murphy, E. A., Faraone, S. V., Chaloff, J., Hirshfeld, D. R. et al. (1993). Behavioral inhibition in childhood: A risk factor for anxiety disorders. Harvard Review of Psychiatry, 1, 2-16.

Rosenbaum, J. F., Biederman, J., Hirshfeld-Becker, D. R., Kagan, J., Snidman, N., Nineberg, A., Gallery, D. y Faraone, S. V. (2000). A controlled study of behavioural inhibition in children of parents with panic disorder and depression. American Journal of Psychiatry, 157, 2002-2010.

Rosenbaum, J. F., Biederman, J., Pollack, R. A. y Hirshfeld, D. R. (1994). The etiology of social phobia. Journal of Clinical Psychiatry, 55, 10-16.

Samuels, J., Nestadt, G., Bienvenu, O. J., Costa, P. T., Riddle, M. A., Liang, K. Y., Hoehn-Saric, R., Grados, M. y Cullen, B. A. M. (2000). Personality disorders and normal personality dimensions in obsessivecompulsive disorder: Results from the Johns Hopkins OCD Family Study. British Journal of Psychiatry, 177, 457-462.

Sanderson, W. C., Rapee, R. M. y Barlow, D. H. (1989). The influence of perceived control on panic attacks induced via inhalation of $5.5 \% \mathrm{CO}_{2}$ enriched air. Archives of General Psychiatry, 46, 157-162.

Schmidt, N. B., Lerew, D. R. y Jackson, R. J. (1997). The role of anxiety sensitivity in the pathogenesis of panic: Prospective evaluation of spontaneous panic attacks during acute stress. Journal of Abnormal Psychology, 106(3), 355-364.

Schmidt, N. B., Lerew, D. R. y Jackson, R. J. (1999). Prospective evaluation of anxiety sensitivity in the pathogenesis of panic: Replication and extension. Journal of Abnormal Psychology, 108, 532-537.

Schmidt, N. B., Trakowski, J. H. y Staab, J. P. (1997). Extinction of panicogenic effects of a $35 \% \mathrm{CO} 2$ challenge in patients with panic disorder. Journal of Abnormal Psychology, 106(4), 630-638. 
Schwartz, C. E., Snidman, N. y Kagan, J. (1999). Adolescent social anxiety as an outcome of inhibited temperament in childhood. Journal of the American Academy of Child \& Adolescent Psychiatry, 38, 1008-1015.

Segui, J., Marquez, M., García, L., Canet, J., Salvador-Carulla, L. y Ortiz, M. (1999). Differential clinical features of early-onset panic disorder. Journal of Affective Disorders, 54, 109-117.

Seivewright, H., Tyrer, P. y Johnson, T. (2004). Persistent social dysfunction in anxious and depressed patients with personality disorder. Acta Psychiatrica Scandinavica, 109, 104-109.

Shafar, S. (1976). Aspects of phobic illness- A study of 90 personal cases. British Journal of Medical Psychology, 49, 221-236.

Simon, N. M., Otto, M. W., Smits, J. A. J., Colette Nicolau, D. Reese, H. E. y Pollack, M. H. (2004). Changes in anxiety sensitivity with pharmacotherapy for panic disorder. Journal of Psychiatric Research, 38, 491-495.

Slaap, B. R. y den Boer, J. A. (2001). The prediction of nonresponse to pharmacotherapy in panic disorder: a review. Depression and Anxiety, 14, 112-122.

Smits, J. A., Powers, M. B., Cho, Y. y Telch, M. J. (2004). Mechanism of change in cognitive-behavioral treatment of panic disorder: Evidence for the fear of fear meditational hypothesis. Journal of Consulting and Clinical Psychology, 72, 646-652.

Sokolowski, K. L. e Israel, A. C. (2008). Perceived anxiety control as a mediator of the relationship between family stability and adjustment. Journal of Anxiety Disorders, 22, 1454-1461.

Starcevic, V., Bogojevic, G., Marinkovic, J. y Kelin, K. (1999). Axis I and Axis II comorbidity in panic/ agoraphobic patients with and with out suicidal ideation. Psychiatry Research, 88, 153-161.

Starcevic, V., Latas, M., Kolar, D., Vucinic-Latas, D., Bogojevic, G. y Milovanovic, S. (2008). Co-ocurrence of axis I disorders in female and male patients with panic disorder with agoraphobia. Comprehensive Psychiatry, 49, 537-543.

Taylor, S., Koch, W. J. y McNally, R. J. (1992). How does anxiety sensitivity vary across the anxiety disorders?. Journal of Anxiety Disorders, 6, 249-259.

Telch, M. J., Jacquin, k., Smits, J. A. y Powers, M. B. (2003). Emotional responding to hyperventilation as a predictor of agoraphobia status among individuals suffering from panic disorder. Journal of Behavior Therapy and Experimental Psychiatry, 34, 161-170.
Telch, M. J., Kamphuis, J. H. y Schmidt, N. B. (2011). The effects of comorbid personality disorders on cognitive behavioral treatment for panic disorder. Journal of Psychiatric Research, 45, 469-474.

Tennant, C., Hurry, J. y Bebbington, P. (1982). The relation of childhood separation experiences to adult depressive and anxiety states. British Journal of Psychiatry, 141, 175-182.

Thorpe, G. L. y Burns, G. J. (1983). The agoraphobic syndrome. New York, John Wiley y Sons.

Uhlenhuth, E. H., Starcevic, V., Warner, T. D., Matuzas, W., McCarty, T., Roberts, B. y Jenkusky, S. (2002). A general anxiety-prone cognitive style in anxiety disorders. Journal of Affective Disorders, 70, 241-249.

Venturello, S., Barzega, G., Maina, G. y Bogetto, F. (2002). Premorbid conditions and precipitating events in early-onset panic disorder. Comprehensive Psychiatry, 43, 28-36.

Watson, D. y Pennebaker, J. (1989). Health complaints, stress and distress: Exploring the control role of negative affectivity. Psychological Review, 96, 234-254.

Webster, A. S. (1953). The development of phobias in married women. Psychological Monographs, 67, 367.

Weems, C. F., Hayward, C., Killen, J. y Taylor, C. B. (2002). A longitudinal investigation of anxiety sensitivity in adolescence. Journal of Abnormal Psychology, 111, 471-477.

White, K. S. y Barlow, D. H. (2002). Panic disorder and agoraphobia. En D. H. Barlow (Ed.), Anxiety and its disorders: the nature and treatment of anxiety and panic (pp.328-379). The Guilford Press, New York. London.

White, K. S., Brown, T. A., Somers, T. J. y Barlow, D. H. (2006). Avoidance behavior in panic disorder: The moderating influence of perceived control. Behaviour Research and Therapy, 44, 147-157.

Zebb, B. J. y Moore, M. C. (2003). Superstitiousness and perceived anxiety control as predictors of psychological distress. Journal of Anxiety Disorders, 17, 115-130.

Zvolensky, M. J., Bernstein, A, Marshall, E. C. y Feldner, M. T. (2006). Panic attacks, panic disorder, and agoraphobia: Associations with substance use, abuse, and dependence. Current Psychiatry Reports, 8, 279-285.

(Artículo recibido: 12-4-2012; revisado: 16-4-2013; aceptado: 20-4-2013) 